\title{
Markov invariants, plethysms, and phylogenetics
}

\author{
J G Sumner ${ }^{1,2}$, M A Charleston ${ }^{1}$, L S Jermiin ${ }^{3}$ and P D Jarvis ${ }^{2, \dagger}$ \\ ${ }^{1}$ School of Information Technologies, University of Sydney \\ ${ }^{2}$ School of Mathematics and Physics, University of Tasmania \\ ${ }^{3}$ School of Biological Sciences, University of Sydney
}

\begin{abstract}
We explore model based techniques of phylogenetic tree inference exercising Markov invariants. Markov invariants are group invariant polynomials and are distinct from what is known in the literature as phylogenetic invariants, although we establish a commonality in some special cases. We show that the simplest Markov invariant forms the foundation of the Log-Det distance measure. We take as our primary tool group representation theory, and show that it provides a general framework for analysing Markov processes on trees. From this algebraic perspective, the inherent symmetries of these processes become apparent, and focusing on plethysms, we are able to define Markov invariants and give existence proofs. We give an explicit technique for constructing the invariants, valid for any number of character states and taxa. For phylogenetic trees with three and four leaves, we demonstrate that the corresponding Markov invariants can be fruitfully exploited in applied phylogenetic studies.
\end{abstract}

\footnotetext{
$\dagger$ Alexander von Humboldt Fellow

keywords: invariants, plethysm, phylogenetics, Markov, time-inhomogeneous, semigroup, generating function, Schur-Weyl duality, Schur functions, branching rules

email: jsumner@it.usyd.edu.au

UTAS-PHYS-2007-31
} 


\section{Contents}

1 Introduction 1

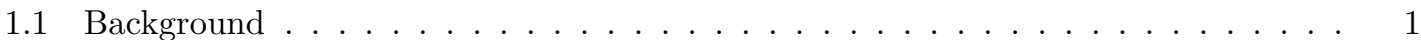

1.2 Markov invariants . . . . . . . . . . . . . . . . . 3

2 Measure theory, the Markov semigroup, and phylogenetic tensors 4

2.1 Probability measures on finite sets . . . . . . . . . . . . 4

2.2 Random variables, generating function, expectation values and estimators . . . . . 5

2.3 The Markov semigroup . . . . . . . . . . . . . . . . . . . . 7

2.4 Phylogenetic tensors . . . . . . . . . . . . . . . . . . . . . . . . . 9

2.5 Markov invariants, definition . . . . . . . . . . . . . . . 12

3 Group representation theory in phylogenetics 13

3.1 The Markov semigroup and affiliated groups . . . . . . . . . . . . . . . . 13

3.2 Representations of $G L(k)$ and Schur-Weyl duality . . . . . . . . . . . . . . . . 14

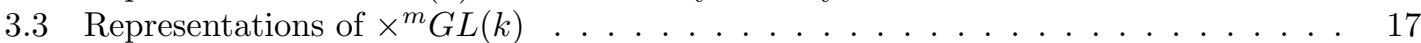

3.4 Symmetric plethysms and invariants . . . . . . . . . . . . . 18

3.5 Markov invariants, existence theorems . . . . . . . . . . . . . . . . . . 19

4 Markov invariants in phylogenetics 2

4.1 Zoo of invariants and nomenclature . . . . . . . . . . . . . . . 21

4.2 What happens on a phylogenetic tree? . . . . . . . . . . . . . . 22

4.3 Mixed weight Markov invariants . . . . . . . . . . . . . . . . 27

5 Discussion 28

A Proof of Theorem 1

B Young's operators and the construction of Markov invariants 32

C Kimura 3ST model and phylogenetic invariants 33

\section{Introduction}

\subsection{Background}

Phylogenetic tree reconstruction aims to infer the past evolutionary relationships of organisms from present day molecular data such as DNA sequences. Progress is made by making astute assumptions about the evolutionary process which simplify the problem into a mathematical form, while retaining much of the structure which motivates the biological question at hand. This process of mathematical modeling is absolutely essential if informed inferences from observed data sets are to be made.

The most significant simplification that is made in phylogenetic models is that the evolutionary change of the molecular units is assumed to progress by mutation under environmental influences and the Darwinian effects of selection are ignored. Another overriding simplification featuring in all the popular models is that the effect of mutations is modelled as a stochastic (random) process assumed to be Markov. Also, it is often assumed that any given site in a molecular sequence evolved independently of the other sites, and the probability of mutation at each site is identically distributed (known together as the IID assumption.) Although the IID assumption is known not to hold in many cases [47, we will assume throughout that IID holds, and defer modification of the results presented here to this more general case.

Much progress in phylogenetic inference has been achieved in recent years with the use of sophisticated mathematics, probability and statistical theory, and the advent of powerful computing techniques. A general rule that rates the scientific credence of a tree reconstruction technique is 
that model based techniques are preferred. In particular, some recent work has focused on the elucidation of the implicit model assumptions of popular techniques of tree reconstruction such as Neighbor-Joining [10, 20] and Maximum Parsimony [71. This type of analysis is an essential part of the scientific justification because otherwise it is not exactly clear what is being estimated in the statistical sense. Without such a framework the biologist is left without any information regarding the confidence in the inference produced.

An overlying difficulty in phylogenetic tree reconstruction is that the number of possible trees is vast, and the space of trees is non-Euclidean; hence it is not clear how one should proceed in searching through it. It is normal to begin with a candidate tree and then consider each of its neighbouring trees (under a given adjacency rule) and choose the new tree as that with the best score function. There is a range of available tree perturbation types such as "prune and regraft" or Nearest Neighbour Interchange which define these adjacencies. Which type is preferable is a matter of ongoing debate [12, 29] and such heuristic techniques are bound to find only locally optimal solutions. In this paper we will not discuss the problems associated with large trees, but consider how small trees may be built with great confidence. We give a general framework for constructing small trees under very general model assumptions which can then be used as a springboard for building larger trees using techniques such as 'quartet puzzling' 65] or supertree methods (for arbitrarily sized subtrees) 9,75 .

Due to its importance for calculating divergence times of lineages, the rate of mutation present in models of evolution is of central importance in phylogenetics. There are several well-known limitations of the standard models involving the rate of mutation on a phylogenetic tree. For instance the IID assumption is almost always violated by the existence of site to site rate variation [55] and by the existence of invariable sites [45. Other issues include non-stationary processes of evolution (which leads to 'compositional heterogeneity' [37]), 'pattern heterogeneity' (where the pattern of substitutions varies across the sites [55]) and 'heterotachy' (differential rates across the tree) [46]. Ignoring the invalidity of the simple models when such assumptions are violated leads to model mis-specification 62 and (potentially) incorrect tree reconstruction.

An issue for any reconstruction technique is that of 'consistency'; where consistency is always with respect to an explicit or implicit model (or family of models) of sequence evolution. Statistical consistency requires that if the data set is sampled from a distribution generated under the model assumptions, then the inference method tends to the correct answer $100 \%$ of the time as the size of the data set (length of the sequences) tends to infinity. For example Felsenstein [16 showed that Maximum Parsimony (MP) is statistically inconsistent (with all but a small family of models 61]).

As exemplified by the first three chapters of the recent review book [19, the statistically consistent, model based phylogenetic reconstruction techniques can placed into three categories: Minimum Evolution (ME) and distance based methods, Maximum Likelihood (ML), and Bayesian methods. ME proceeds by defining a (model based) matrix of pairwise distances between the molecular sequences, and then minimizes the total tree length across the space of possible trees subject to some statistical criteria such as least squares (see Chapter 1 of [19].) ML proceeds by maximizing the 'likelihood' of the observed data set across the set of possible trees and models of evolution [17, 19]. Bayesian methods proceed using Bayes' theorem to calculate a posterior distribution on the space of possible trees given a prior distribution (usually uniform-which is an issue in itself as this does not correspond to any evolutionary model of tree generation 79 .) For each of these techniques the underlying model assumptions are explicit, and current research efforts revolve around implementing these techniques under expanded assumptions and/or in a computationally efficient manner.

Another desirable feature of any reconstruction technique is that the model on which it is based should be defined by as few numerical parameters as possible. The issue of scientific content of a model and parameter counts is discussed by Steel 62 in relation to the effectiveness of MP vs ML, where it was stated that the "predictive power of the theory... tends to be drowned out in a sea of parameter estimation." This is a fundamental problem in model selection for biological inference, and corresponds to what is known as the bias/variance trade off of parameter estimation [11; which in turn equates to the problem of "overfitting" or "underfitting" a data set. From an 
information theoretic perspective, a given data set contains only so much information from which the numerical parameters of a model may be estimated. A model with many parameters may fit the data very well in that the parameter estimates may be close to their true values, but the corresponding variances will be large because there are relatively few data points. On the other hand, the variance of the estimates of a model with very few parameters will be smaller as there are many data points to estimate each parameter, but in this case the model runs the risk of being badly mis-specified so that the parameter estimates may be biased. In this light, the 'covarion' model [56] deals with the effects of invariable sites whilst introducing only one extra parameter, and the 'gamma' model [78 accounts for site to site rate variation, with only an additional two parameters. Other methods for coping with heterotachy, rate variation and pattern heterogeneity include the partitioning of data sets and mixture models [55. However, all of these methods suffer because, in the general case, the models must include an individual rate matrix (containing up to twelve parameters) and an edge length parameter for each and every edge of the phylogenetic tree. In [57] it was recently noted that the task of phylogenetic tree reconstruction often lies in a region where there are more parameters than data points.

To reduce the number of parameters in these models, the evolutionary process is usually assumed to be stationary (the frequency of each DNA base in the sequence is constant in time), the rate matrices are assumed to have a certain form (such as the Jukes-Cantor model with one parameter, or the Kimura model with two or three parameters), and each edge is assigned with the identical rate matrix. To accommodate non-stationary processes and associated compositional heterogeneity, it becomes necessary to introduce many more parameters into the model. In this circumstance it then becomes desirable to use a technique based on a general model but without the need to estimate the numerical parameters. In this light, a matrix of Log-Det pairwise distances combined with the Neighbor-Joining algorithm [48 achieves statistically consistent tree reconstruction under the assumption of a general model. However this technique has its own shortcomings as distance methods only consider pairwise sequence alignments, ignoring much of the information available in the data set, and has problems with model mis-specification [70, and the statistical properties of the Log-Det are not exactly known [23. A recently presented method 35] fits a very general model, but clearly will have issues with over-parameterization and computational requirements.

In summary, the desirable features of a given phylogenetic tree reconstruction technique are that it is based on a general model of sequence evolution, it is statistically consistent with a family of known models, and the number of parameters to be estimated is minimal.

\subsection{Markov invariants}

In this work we introduce the use of mathematical representation theory to the problem of phylogenetic tree reconstruction (further background to the results is presented in the $\mathrm{PhD}$ thesis 68 .) We define 'Markov invariants' and show that these functions, when evaluated on sequence data, can be put to work in the problem of phylogenetic tree reconstruction under rather general model assumptions.

Markov invariants are distinct from what is known in the literature as 'phylogenetic invariants' [1, 2, 24, 64. The definition of a Markov invariant is that of a group invariant function [54]. This is more constraining than the definition of a phylogenetic invariant. A Markov invariant is sometimes simultaneously a phylogenetic invariant, but the reverse is not true in general. The structure of Markov invariants are more akin to that of the Log-Det function [48, which is the simplest example of a Markov invariant, yet is not a phylogenetic invariant (see 68, for more details.)

The desirable feature of this approach is that Markov invariants do not assume any particular rate matrices or edge length parameters on the phylogenetic tree. Broad conditions of molecular evolution are thus accommodated, incorporating arbitrary substitution rates, non-stationary and time-inhomogeneous processes, heterotachy, and arbitrary pattern heterogeneity across the tree. Further, Markov invariants satisfy certain algebraic relations for particular phylogenetic trees, and can provide a novel method of tree inference. 
This approach to phylogenetic tree inference satisfies the desirable features given in the summary above. That is, Markov invariants are valid for a general model of sequence evolution, statistical consistency is assured, and only a few parameters need to be estimated.

In particular, for the quartet case, we give a tree inference routine, valid for these inclusive conditions, optimizing over only two parameters . It is hoped that, with additional understanding, this technique can be extended to larger trees. This will result in phylogenetic tree inference methods, valid for general models, that make use of only a few parameters. Such a possibility is very attractive, as all of the data is utilized, and a general model may be assumed with the risk of overfitting significantly reduced.

In this paper we outline the theoretical background required to understand the derivation of Markov invariants. This will necessitate, in $\$ 2$, an excursion into elementary measure theory on finite sets, and the construction of 'phylogenetic tensors'. In \$3 we analyse certain groups affiliated with the Markov process, and review standard results from group representation theory. This section concludes with a derivation of existence conditions for Markov invariants. In 44 we report on the structure of Markov invariants for phylogenetic trees with three and four leaves, and give examples of how they can be incorporated into practical phylogenetic analyses.

\section{Measure theory, the Markov semigroup, and phylogenetic tensors}

In $\$ 2.1$ we collect some basic properties of measures on finite sets, justifying the use of tensor product spaces in the context of Markov processes on phylogenetic trees. The results are rather elementary, but ultimately necessary to place the subsequent discussion on its proper footing. See, for example, 25. for an introduction to measure theory. In 2.2 we use generating function techniques to calculate expectation values of various random variables (and functions thereof) associated with phylogenetic data sets. We give a simple example and show how to compute its unbiased estimator. We define the 'Markov semigroup' for the general time-inhomogeneous process (\$2.3), construct 'phylogenetic tensors' (\$2.4), and, finally, define Markov invariants (\$2.5).

\subsection{Probability measures on finite sets}

Consider a finite set labelled by natural numbers, $K=\{1,2, \ldots, k\}$. A probability measure on $K$, is a function $\mu: K \rightarrow[0,1]$, such that, for any proper subset $A \subset K$ and any sequence $A_{1}, A_{2}, \ldots$ of pairwise disjoint subsets, the following conditions hold:

$$
\begin{aligned}
\mu(\varnothing) & =0, \\
\mu(A) & <1, \\
\mu\left(\bigcup_{i} A_{i}\right) & =\sum_{i} \mu\left(A_{i}\right), \\
\mu(K) & =1 .
\end{aligned}
$$

We denote the set of probability measures on $K$ as $\mathcal{M}(K)$. It follows from the third condition that for $1 \leq i \leq k$ the measures, $\delta_{i}(A)=1$ if $i \in A$ and 0 otherwise, form a basis such that

$$
\mu=\sum_{i=1}^{k} \mu_{i} \delta_{i},
$$

for all $\mu \in \mathcal{M}(K)$ with $\mu_{i}:=\mu(\{i\})$. This definition is equivalent to the usual requirement of a probability distribution on a finite set:

$$
\sum_{i=1}^{k} \mu_{i}=\mu\left(\bigcup_{i=1}^{k}\{i\}\right)=\mu(K)=1 .
$$


In phylogenetics the data sets under consideration are aligned sequences of molecular units. For example, in the case of DNA sequences made up of the four nucleotides adenine, cytosine, guanine, thymine, we would have $K=\{A, C, G, T\}, k=4$ and write $K=\{1,2,3,4\}$. However, the results presented here and in $\$ 3$ are valid for any $k$. In $\$ 4$ we will concentrate on cases relevant to phylogenetics and investigate the Markov invariants for $k=2,3$ and 4 .

In this work we do not consider the problem of aligning the sequence data, and assume throughout that the 'true' alignment can and has been found (where truth is relative to the modelling process.) Under this circumstance, it becomes necessary to consider the direct product of $K$ with itself $m$ times:

$$
K^{m}:=\times^{m} K=K \times K \times \ldots \times K
$$

with $\left|K^{m}\right|=k^{m}$. Exactly as above, for any proper subset $E \subset K^{m}$ and any sequence of pairwise disjoint subsets $E_{1}, E_{2}, \ldots$, a probability measure, $\mu \in \mathcal{M}\left(K^{m}\right)$, must equivalently satisfy

$$
\begin{aligned}
\mu(\varnothing) & =0, \\
\mu(E) & <1, \\
\mu\left(\bigcup_{i} E_{i}\right) & =\sum_{i} \mu\left(E_{i}\right), \\
\mu\left(K^{m}\right) & =1 .
\end{aligned}
$$

Given that under a measure unions decompose into summations, it follows that we have the tensor product:

$$
\mathcal{M}\left(K^{m}\right)=\otimes^{m} \mathcal{M}(K):=\mathcal{M}(K) \otimes \mathcal{M}(K) \otimes \ldots \otimes \mathcal{M}(K) .
$$

Concretely, any subset of $K^{m}$ can be expressed as a union of disjoint subsets of the form

$$
A_{1} \times A_{2} \times \ldots \times A_{m},
$$

with $A_{1}, A_{2}, \ldots, A_{m} \subseteq K$. A basis for $\otimes^{m} \mathcal{M}(K)$ is then, for $1 \leq i_{1}, i_{2}, \ldots, i_{m} \leq k$,

$$
\delta_{i_{1}} \otimes \delta_{i_{2}} \otimes \ldots \otimes \delta_{i_{m}}\left(A_{1} \times A_{2} \times \ldots \times A_{m}\right):=\delta_{i_{1}}\left(A_{1}\right) \delta_{i_{2}}\left(A_{2}\right) \ldots \delta_{i_{m}}\left(A_{m}\right),
$$

with $\delta_{i_{1}}\left(A_{1}\right) \delta_{i_{2}}\left(A_{2}\right) \ldots \delta_{i_{m}}\left(A_{m}\right)=1$ if $\left\{i_{1}\right\} \times\left\{i_{2}\right\} \times \ldots \times\left\{i_{m}\right\} \in A_{1} \times A_{2} \times \ldots \times A_{m}$ and 0 otherwise. We index the elements $\left\{i_{1}\right\} \times\left\{i_{2}\right\} \times \ldots \times\left\{i_{m}\right\}$ as

$$
I=i_{1} i_{2} \ldots i_{m}
$$

and write

$$
\mu_{I} \equiv \mu_{i_{1} i_{2} \ldots i_{m}}:=\mu\left(\left\{i_{1}\right\} \times\left\{i_{2}\right\} \times \ldots \times\left\{i_{m}\right\}\right) .
$$

We refer to $m$ as the rank of the tensor $\mu$.

Previously the authors JGS and PDJ have presented probability distributions on phylogenetic trees in a tensor product formalism motivated from analogies to quantum physics [34, 69]. The formulation presented above places this construction on its proper measure-theoretic footing. In 2.4 we will relate a given (Markov) model of evolution on a phylogenetic tree with $m$ leaves, to a unique rank- $m$ tensor $P \in \otimes^{m} \mathcal{M}(K)$.

\subsection{Random variables, generating function, expectation values and es- timators}

Any data set considered in a phylogenetic study is necessarily of finite extent, and we suppose that it is a sample drawn from some unknown distribution. We wish to define expectation values

\footnotetext{
${ }^{1}$ We are indebted to Michael Baake for drawing our attention to this.
} 
of such data (or events) and functions thereof. Throughout we will assume the IID assumption holds, so that we need only consider the distribution of a single random variable. The probability of observing a particular state at a given site will be identical for all the other sites.

For a set of $m$ aligned sequences of length $N$, define a pattern to be the (ordered) set of states read across the $m$ sequences at a particular site in the alignment. That is, a pattern takes the form $I=i_{1} i_{2} \ldots i_{m}$, where $i_{a}$ is the character state in the $a^{t h}$ sequence. Define the random variable $X$ as the pattern observed at a given site. A probability distribution for $X$ can be specified using a probability measure $\mu \in \otimes^{m} \mathcal{M}(K)$ :

$$
\mathbb{P}\left[X=i_{1} i_{2} \ldots i_{m}\right]=\mu_{i_{1} i_{2} \ldots i_{m}} .
$$

For a sequence of finite length $N$, define $Z$ as the random variable that counts the number of occurrences of each pattern $I=i_{1} i_{2} \ldots i_{m}$ in the alignment, so that

$$
Z=\left(Z_{I}\right)=\left(Z_{i_{1} i_{2} \ldots i_{m}}\right)_{1 \leq i_{1}, i_{2}, \ldots, i_{m} \leq k}
$$

and $\sum_{I \in K^{m}} Z_{I}=N$. Assuming that each site in the alignment is identically and independently distributed as (11), it follows that $Z$ is multinomially distributed under the measure $\mu$ :

$$
\mathbb{P}[Z=z ; N]=\prod_{I \in K^{m}} \frac{N !}{z_{I} !} \mu_{I}^{z_{I}} .
$$

This expresses, under the assumptions of $\mu$, the probability of observing within the alignment of $m$ sequences the specific number of occurrences of each of the possible character patterns $Z=z$.

When we describe Markov invariants, we will need to discuss expectation values of the random variable $Z$ and functions thereof. For any function $\phi$, the expectation value with respect to the measure $\mu$ is defined as

$$
E[\phi(Z)]:=\sum_{z} \phi(z) \mathbb{P}[Z=z ; N]
$$

with the summation over all $z$ such that $\sum_{I \in K^{m}} z_{I}=N$.

Remembering that $Z$ follows a multinomial distribution, it is in practice necessary to use generating function techniques in order to calculate these expectation values. The generating function on the formal variables $s=\left(s_{I}\right)=\left(s_{i_{1} i_{2} \ldots i_{m}}\right)_{1 \leq i_{1}, i_{2}, \ldots, i_{m} \leq k}$ of the multinomial distribution is

$$
G(s):=E\left[e^{(s, Z)}\right]=\left(\sum_{I \in K^{m}} \mu(I) e^{s_{I}}\right)^{N}
$$

with

$$
(s, Z):=\sum_{I \in K^{m}} s_{I} Z_{I}
$$

From the properties of the exponential function and the commutivity of differentiation and expectation,

$$
\left.\frac{\partial G(s)}{\partial s_{i_{1} i_{2} \ldots i_{m}}}\right|_{s=0}=E\left[Z_{i_{1} i_{2} \ldots i_{m}}\right] .
$$

Using the above closed form of the generating function, an elementary calculation returns

$$
E\left[Z_{i_{1} i_{2} \ldots i_{m}}\right]=N \mu_{i_{1} i_{2} \ldots i_{m}},
$$

as of course would be expected. This can be extended to find the expectation of any function of $Z$ :

$$
E[\phi(Z)]=\left.\phi\left(\frac{\partial}{\partial s}\right) G(s)\right|_{s=0} .
$$


As a concrete example, take $m=2$ and consider the case $\phi(Z)=Z_{44}^{2}-Z_{12} Z_{13}$. From the linearity of the expectation values we have

$$
E\left[Z_{44}^{2}-Z_{12} Z_{13}\right]=E\left[Z_{44}^{2}\right]-E\left[Z_{12} Z_{13}\right],
$$

so we can consider each term in turn. Taking derivatives of the closed form of the generating function gives

$$
E\left[Z_{44}^{2}\right]=N(N-1) \mu_{44}^{2}+N \mu_{44}
$$

and

$$
E\left[Z_{12} Z_{13}\right]=N(N-1) \mu_{12} \mu_{13} .
$$

Thus, in this case, the expectation value of $\phi$ is

$$
E[\phi(Z)]=N(N-1)\left(\mu_{44}^{2}-\mu_{12} \mu_{13}\right)+N \mu_{44} .
$$

Given a (possibly unobservable) random variable $\theta$, an estimator is another random variable which is a function of observable quantities such that its expectation value somehow approximates $\theta$. The bias of an estimator $\widehat{\theta}$ is defined as the difference

$$
b(\hat{\theta})=E[\hat{\theta}]-E[\theta],
$$

allowing for $\theta$ to simply be a constant so that $E[\theta]=\theta$. An unbiased estimator is simply an estimator with bias equal to zero. For example, a short calculation reveals that the unbiased estimator of $\phi(\mu)$ above is

$$
\frac{\phi(Z)-Z_{44}}{N(N-1)}
$$

In general, if $\phi$ is polynomial, computing an unbiased form is a straightforward matter of solving a sequence of difference equations. When it comes to discussing estimators for Markov invariants, we will show that unbiased forms can easily be defined. However, we will note that explicit computation is difficult due to a required change of basis.

\subsection{The Markov semigroup}

A stochastic process can be described by introducing a time-dependent random variable $X(t)$. A crucial component of the subsequent discussion will be that the time evolution of the corresponding probability distribution can be viewed as a linear mapping upon a vector space. Presently we will establish the conditions for a Markov process, and show that such a process satisfies the desired property. See, for example, 32 for an equivalent derivation.

Consider a time-dependent, finite-state random variable, $X(t)$, taking on values in $K$, any set of times $t_{1}<t_{2}<\ldots<t_{n}<t$, and the joint distribution of $X$ across those times:

$$
\mathbb{P}\left[X\left(t_{1}\right)=i_{1}, X\left(t_{2}\right)=i_{2}, \ldots, X\left(t_{n}\right)=i_{n}, X(t)=i\right] .
$$

The distribution of $X$ at the particular time $t$ is given by the marginal,

$$
\mathbb{P}[X(t)=i]=\sum_{1 \leq i_{1}, i_{2}, \ldots, i_{n} \leq k} \mathbb{P}\left[X\left(t_{1}\right)=i_{1}, X\left(t_{2}\right)=i_{2}, \ldots, X\left(t_{n}\right)=i_{n}, X(t)=i\right],
$$

and this can be re-expressed by invoking the conditional distribution:

$$
\begin{array}{r}
\mathbb{P}[X(t)=i]=\sum_{1 \leq i_{1}, i_{2}, \ldots, i_{n} \leq k} \mathbb{P}\left[X(t)=i \mid X\left(t_{1}\right)=i_{1}, X\left(t_{2}\right)=i_{2}, \ldots, X\left(t_{n}\right)=i_{n}\right] \\
\cdot \mathbb{P}\left[X\left(t_{1}\right)=i_{1}, X\left(t_{2}\right)=i_{2}, \ldots, X\left(t_{n}\right)=i\right] .
\end{array}
$$


The simplest stochastic process is the process for which the probability of a transition to a new state at a given time is independent of the states at all preceding times (such as tossing of a coin-the Bernoulli process.) A Markov process can be seen as the next simplest case where the probability of a transition is independent of all but the state at the most recent time. Thus, for a Markov process the conditional distribution satisfies

$$
\mathbb{P}\left[X(t)=i \mid X\left(t_{1}\right)=i_{1}, X\left(t_{2}\right)=i_{2}, \ldots, X\left(t_{n}\right)=i_{n}\right]=\mathbb{P}\left[X(t)=i \mid X\left(t_{n}\right)=i_{n}\right] .
$$

This implies that the marginal distribution of $X$ at the time $t$ is

$$
\begin{aligned}
& \mathbb{P}[X(t)=i]=\sum_{1 \leq i_{n} \leq k} \mathbb{P}\left[X(t)=i \mid X\left(t_{n}\right)=i_{n}\right] \\
& \cdot \sum_{1 \leq i_{1}, \ldots, i_{n-1} \leq k} \mathbb{P}\left[X\left(t_{1}\right)=i_{1}, X\left(t_{2}\right)=i_{2}, \ldots, X\left(t_{n}\right)=i_{n}\right] \\
&=\sum_{1 \leq i_{n} \leq k} \mathbb{P}\left[X(t)=i \mid X\left(t_{n}\right)=i_{n}\right] \mathbb{P}\left[X\left(t_{n}\right)=i_{n}\right] .
\end{aligned}
$$

Introducing the time-dependent measure $\mu^{t}$ with $\mu^{t}(\{i\}):=\mu_{i}^{t}=\mathbb{P}[X(t)=i]$, we can express this as

$$
\mu_{i}^{t}=\sum_{1 \leq j \leq k} M_{i j}(t, s) \mu_{j}^{s}
$$

for all $s<t$, and for $M_{i j}(t, s):=\mathbb{P}[X(t)=i \mid X(s)=j]$. If we consider the $\left(M_{i j}(t, s)\right)_{1 \leq i, j \leq k}$ as the matrix elements of a linear operator $M(t, s)$ acting on the vector space $\mathbb{R}^{k} \supset \mathcal{M}(K)$ with basis elements $\delta_{1}, \delta_{2}, \ldots, \delta_{k}$, we see that, as promised, for a Markov process the time evolution of the probability distribution is given by a linear map on $\mathbb{R}^{k}$ defined by its action on time-dependent probability measures:

$$
\begin{aligned}
& \mu^{s} \stackrel{M(t, s)}{\mapsto} \mu^{t}, \\
& \mu^{t}=M(t, s) \mu^{s} .
\end{aligned}
$$

This linear map describes the general time-inhomogeneous finite state Markov process and can easily be extended to the whole of $\mathbb{R}^{k}$.

In [69] JGS and PDJ considered stochastic matrices as elements of the general linear group, and used this property to study the structure of invariant polynomials (used as measures of entanglement in quantum physics) when evaluated on a phylogenetic tree. Presently we will define the Markov semigroup which serves to refine the definition of invariant functions to the more relevant case of a stochastic (but linear) time evolution.

Define the time-dependent rate matrix, $Q(t)$, as a (continuous) one-parameter family of linear operators on the vector space $\mathcal{M}(K)$, which in the $\delta_{1}, \delta_{2}, \ldots, \delta_{k}$ basis has matrix elements satisfying:

$$
Q_{i j}(t) \geq 0, \quad \forall i \neq j ; \quad Q_{i i}(t)=-\sum_{j \neq i} Q_{j i}(t)
$$

The summation conditions can be equivalently expressed by defining the vector $\theta=\delta_{1}+\delta_{2}+\ldots+\delta_{k}$ and its transpose $\theta^{\top}$, and setting

$$
\theta^{\top} Q(t)=0
$$

for all $t$.

The Markov semigroup on $k$ elements, $\mathfrak{M}(k)$, with parameters $0 \leq s \leq t<\infty$, is defined as the subset of (differentiable) two-parameter linear operators on $\mathcal{M}(K)$ which satisfy

$$
M(t, s)=1, \quad \forall t=s
$$


the Chapman-Kolmogorov equation:

$$
M(t, s) M(s, r)=M(t, r), \quad \forall r<s
$$

and the backwards and forwards equations:

$$
\begin{aligned}
& \frac{\partial M(t, s)}{\partial s}=-M(t, s) Q(s), \\
& \frac{\partial M(t, s)}{\partial t}=Q(t) M(t, s) ;
\end{aligned}
$$

for any rate matrix $Q(t)$ [21, 32]. Solutions of (3) can be represented using the time-ordered product (or ordered-exponential):

$$
M(t, s)=\mathbb{T} \exp \int_{s}^{t} Q(u) d u
$$

[33], from which it follows that

$$
\operatorname{det} M(t, s)=\exp \int_{s}^{t} \operatorname{tr}(Q(u)) d u
$$

and

$$
\theta^{\top} M(t, s)=\theta^{\top}
$$

The time-ordered product is best understood by considering the approximation

$$
M(s+2 \epsilon, s)=M(s+2 \epsilon, s+\epsilon) M(s+\epsilon, s) \simeq e^{Q(s+\epsilon) \epsilon} e^{Q(s) \epsilon} .
$$

By considering (3) for the case $t=s$, it follows that in the $\delta_{1}, \delta_{2}, \ldots, \delta_{k}$ basis, the matrix elements of each $M(t, s)$ lie in the interval $[0,1]$ for all $s \leq t$. Thus, the Markov semigroup corresponds to the subset of the set of stochastic matrices subject to the condition that for each matrix there exists a rate matrix (or generator) $Q(t)$ such that (4) is satisfied. We refer to elements of the Markov semigroup as Markov operators.

In the time-homogeneous case where the rate matrix is time-independent:

$$
Q:=Q(t)=Q(0),
$$

it follows that $M(t, s)$ is dependent only upon the difference $(t-s)$, and form (4) becomes simply

$$
M(t)=e^{t Q}=\sum_{0 \leq n<\infty} \frac{(t Q)^{n}}{n !} .
$$

In $₫ 3$ we will discuss some representation-theoretic properties of certain groups affiliated with the Markov semigroup.

\subsection{Phylogenetic tensors}

A tree, $\mathcal{T}$, is a connected graph without cycles and consists of a set of vertices and edges. Vertices of degree one are called leaves. We work with oriented trees, which are defined by directing each edge of $\mathcal{T}$ away from a distinguished vertex, $\rho$, known as the root of the tree. Consequently, a given edge lying between adjacent vertices $u$ and $v$ is specified as an ordered pair $(u, v)$, where $u$ lies on the unique path from $\rho$ to $v$. A cherry is a pair of leaf vertices with the same parent vertex.

Assign a random variable, $X_{v}$, to each vertex of the tree, and, as described in [59, a joint distribution of the random variables at the leaves is determined by specifying a distribution $\pi \in$ $\mathcal{M}(K)$ at $\rho$ and a Markov operator $M^{v, u} \in \mathfrak{M}(k)$ for every edge $(u, v)$. In particular, for every 


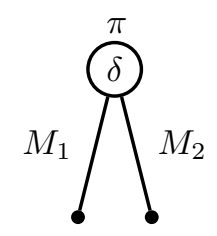

Figure 1: Phylogenetic tree with two leaves

$v$, the random variable $X_{v}$ is conditional upon only the random variables lying on the path from $\rho$ and $v$, and for each pair of vertices $v_{1}, v_{2}$ with common parent $u$, the joint distribution of $X_{v_{1}}$ and $X_{v_{2}}$ is given by

$$
\mathbb{P}\left[X_{v_{1}}=i_{1}, X_{v_{2}}=i_{2}\right]=\sum_{1 \leq j \leq k} M_{i_{1} j}^{v_{1}, u} M_{i_{2} j}^{v_{2}, u} \mu_{j}^{u},
$$

where $\mu^{u}$ is the distribution of $X_{u}$. The empirical interpretation of the joint distribution across the leaves is that of a sampling distribution from which an of alignment molecular sequences is constructed by drawing one character pattern at a time. In the case that the Markov operators and the root distribution are arbitrary, the resulting family of distributions is known as the general Markov model of a phylogenetic tree [1].

We will consider a joint probability distribution on $m$ leaves as a probability measure, $P \in$ $\otimes^{m} \mathcal{M}(K)$, that we refer to as a phylogenetic tensor. Presently we review how these tensors can be constructed using purely algebraic operations.

The branching process (6) can be interpreted as a map that takes probability measures on $K$ to probability measures on $K \times K=K^{2}$. In [34, 69] JGS and PDJ showed how to formalize this by defining the linear operator

$$
\delta: \mathcal{M}(K) \rightarrow \mathcal{M}(K) \otimes \mathcal{M}(K)
$$

Demanding the conditional dependencies that are required for the standard definition of a tree distribution [59], we have (expressed in the $\delta_{1}, \delta_{2}, \ldots, \delta_{k}$ basis) the specification:

$$
\delta: \delta_{i} \mapsto \delta_{i} \otimes \delta_{i}, \quad 1 \leq i \leq k .
$$

The phylogenetic tree with two leaves (Figure 1) can then be represented as the string

$$
P=\left(M_{1} \otimes M_{2}\right) \cdot(\delta \cdot \pi)
$$

where $M_{1}, M_{2}$ are the Markov operators on the two edges of the tree and, if $X_{1}, X_{2}$ are the random variables at the leaves 1 and 2 respectively, we have

$$
\mathbb{P}\left[X_{1}=i, X_{2}=j\right]=P_{i j},
$$

with $P_{i j}:=P(\{i\} \times\{j\})$.

This construction can be generalized to any phylogenetic tree by colouring the root of the tree with a distribution $\pi$, each internal vertex (including the root) with a branching operator $\delta$, and every edge with an arbitrary Markov operator. The phylogenetic tensor is constructed by beginning at the root of the tree, and then recursively moving to the child vertices and applying the relevant operators to the corresponding slots in the (growing) tensor. Whenever a leaf is encountered, continually apply the identity operator at that leaf, until all leaves have been reached and the phylogenetic tensor is complete. A phylogenetic tensor, $P$, is then represented as a string made up of the characters $\pi, M_{1}, M_{2}, \ldots$, and $\delta$, and the joint distribution of the random variables $X_{1}, X_{2}, \ldots, X_{m}$ at the leaves $1,2, \ldots, m$ is given by

$$
\mathbb{P}\left[X_{1}=i_{1}, X_{2}=i_{2}, \ldots, X_{m}=i_{m}\right]=P_{i_{1} i_{2} \ldots i_{m}},
$$

with $P_{i_{1} i_{2} \ldots i_{m}}:=P\left(\left\{i_{1}\right\} \times\left\{i_{2}\right\} \times \ldots \times\left\{i_{m}\right\}\right)$. 


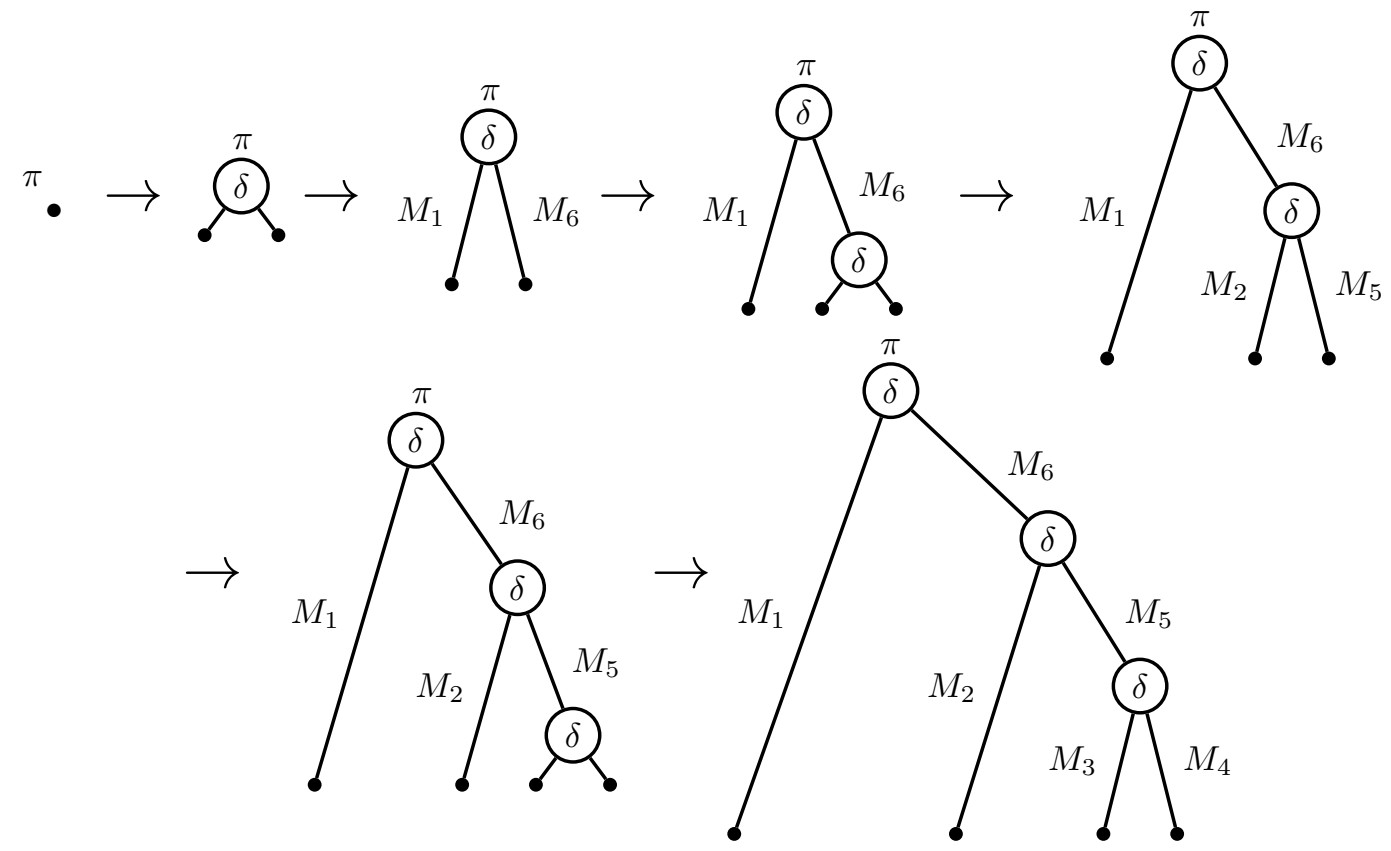

Figure 2: Constructing the phylogenetic tensor for a four taxon tree

For example, the phylogenetic tensor of four leaves (Figure 2) is represented by the string

$$
P=\left(1 \otimes 1 \otimes M_{3} \otimes M_{4}\right) \cdot(1 \otimes 1 \otimes \delta) \cdot\left(1 \otimes M_{2} \otimes M_{5}\right) \cdot(1 \otimes \delta) \cdot\left(M_{1} \otimes M_{6}\right) \cdot(\delta \cdot \pi),
$$

that is constructed in the steps

$$
\begin{aligned}
\pi \rightarrow \delta \cdot \pi & \rightarrow\left(M_{1} \otimes M_{6}\right) \cdot(\delta \cdot \pi) \rightarrow(1 \otimes \delta) \cdot\left(M_{1} \otimes M_{6}\right) \cdot(\delta \cdot \pi) \\
\rightarrow & \left(1 \otimes M_{2} \otimes M_{5}\right) \cdot(1 \otimes \delta) \cdot\left(M_{1} \otimes M_{6}\right) \cdot(\delta \cdot \pi) \\
\quad & (1 \otimes 1 \otimes \delta) \cdot\left(1 \otimes M_{2} \otimes M_{5}\right) \cdot(1 \otimes \delta) \cdot\left(M_{1} \otimes M_{6}\right) \cdot(\delta \cdot \pi) \\
& \rightarrow\left(1 \otimes 1 \otimes M_{3} \otimes M_{4}\right) \cdot(1 \otimes 1 \otimes \delta) \cdot\left(1 \otimes M_{2} \otimes M_{5}\right) \cdot(1 \otimes \delta) \cdot\left(M_{1} \otimes M_{6}\right) \cdot(\delta \cdot \pi) .
\end{aligned}
$$

In order to define Markov invariants, we must also define two reduced tensors based on $P$, the trimmed tensor $\widetilde{P}$ and the pruned tensor $P^{*}$. These are both constructed by modifying the underlying tree. The trimmed tensor $\widetilde{P}$ is constructed by taking $P$ and setting the Markov operators on the pendant edges all equal to the identity operator, or equivalently setting the lengths of the pendant edges to zero. The pruned tensor $P^{*}$ is constructed by removing all cherries from the trimmed tensor. The rank of the pruned tensor is $(m-c)$ where $c$ is the number of cherries on the underlying tree.

In the general case, we see that we can relate $P$ and $\widetilde{P}$ as

$$
P=\left(M_{1} \otimes M_{2} \otimes \ldots \otimes M_{m}\right) \cdot \widetilde{P},
$$

where $M_{1}, M_{2}, \ldots, M_{m}$ are the Markov operators on the leaf edges. In what is to come, we will continually use this relation.

As an illustrative example of the relationship between $P, \widetilde{P}$ and $P^{*}$, take the 7 leaf tree (Figure 3), with phylogenetic tensor given by

$$
\begin{aligned}
P=\left(1 \otimes M_{2} \otimes M_{3} \otimes M_{4} \otimes M_{5} \otimes M_{6} \otimes M_{7}\right) \cdot(1 \otimes \delta \otimes \delta \otimes \delta) \\
\cdot\left(M_{1} \otimes M_{8} \otimes M_{9} \otimes M_{10}\right) \cdot(\delta \otimes \delta) \cdot\left(M_{11} \otimes M_{12}\right) \cdot(\delta \cdot \pi) .
\end{aligned}
$$

The trimmed tensor corresponding to the tree (Figure 44) is obtain by clipping off the pendant edges:

$$
\widetilde{P}=(1 \otimes \delta \otimes \delta \otimes \delta) \cdot\left(1 \otimes M_{8} \otimes M_{9} \otimes M_{10}\right) \cdot(\delta \otimes \delta) \cdot\left(M_{11} \otimes M_{12}\right) \cdot(\delta \cdot \pi),
$$


and, finally the pruned tensor corresponding to the tree (Figure 5) is expressed as:

$$
P^{*}=\left(M_{8} \otimes M_{9} \otimes M_{10}\right) \cdot(1 \otimes \delta) \cdot\left(M_{11} \otimes M_{12}\right) \cdot(\delta \cdot \pi) .
$$

\subsection{Markov invariants, definition}

With the form (7) in mind, we define a Markov invariant of weight $\left(w_{1}, w_{2}, \ldots, w_{m}\right)$ as a function satisfying

$$
f(P)=\left(\operatorname{det} M_{1}\right)^{w_{1}}\left(\operatorname{det} M_{2}\right)^{w_{2}} \ldots\left(\operatorname{det} M_{m}\right)^{w_{m}} f(\widetilde{P}),
$$

for all $M_{1}, M_{2}, \ldots, M_{m} \in \mathfrak{M}(k)$. We exclusively consider polynomial functions, and where $w_{1}=$ $w_{2}=\ldots=w_{m} \equiv w$, the Markov invariant is said to be of weight $w$.

Considering the above discussion of unbiased estimators of random variables, an unbiased estimator of a Markov invariant is a function, $\widehat{f}$, such that

$$
E[\widehat{f}(Z)]=f(P)=\left(\operatorname{det} M_{1}\right)^{w_{1}}\left(\operatorname{det} M_{2}\right)^{w_{2}} \ldots\left(\operatorname{det} M_{m}\right)^{w_{m}} f(\widetilde{P}) .
$$

Such an estimator depends, up to the multiplicative scaling factor, only upon the internal structure of the phylogenetic tree. It is exactly this property that can be productively engaged in the context of phylogenetic tree reconstruction.

Conversely, a phylogenetic invariant for the general Markov model is a function satisfying $f(P) \equiv 0$ for all $P$ belonging to the family of phylogenetic tensors arising from a particular tree (or subset of trees). In $\$$ we will show that there exist Markov invariants for trees with three and four leaves that are simultaneously phylogenetic invariants.

Given a Markov invariant, $f$, consider the induced function, $f^{*}$, defined by

$$
f^{*}\left(P^{*}\right)=f(\widetilde{P}) .
$$

This induced function is easily extended to be defined upon all of $\otimes^{m-c} \mathcal{M}(K)$, where $c$ is the number of cherries on the underlying tree of $P$. Such cases are of special interest for phylogenetic problems. In $\$ 4$ we will review a case (reported in a less general context in [70]) where this induced function is itself a Markov invariant. We suspect that future investigations of Markov invariants will reveal more cases such as this.

In $\$ 3$ we will establish existence conditions for Markov invariants using standard results from group representation theory.

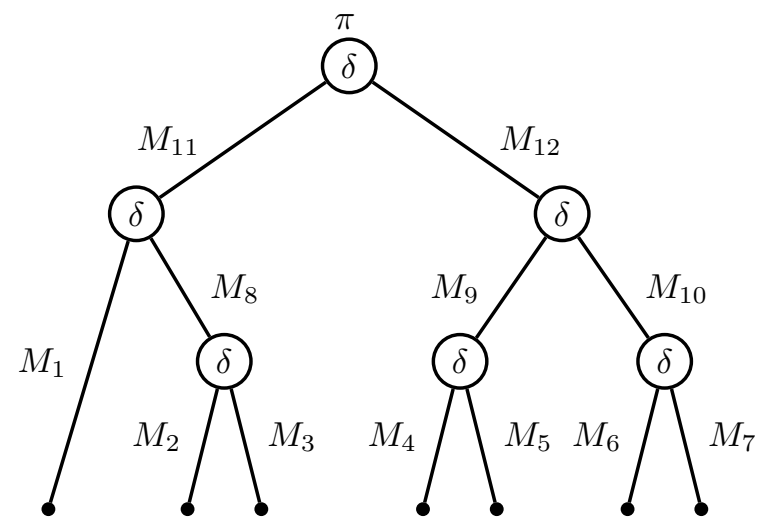

Figure 3: Phylogenetic tensor $P$ for seven taxon tree $((1,23),(45,67))$ 


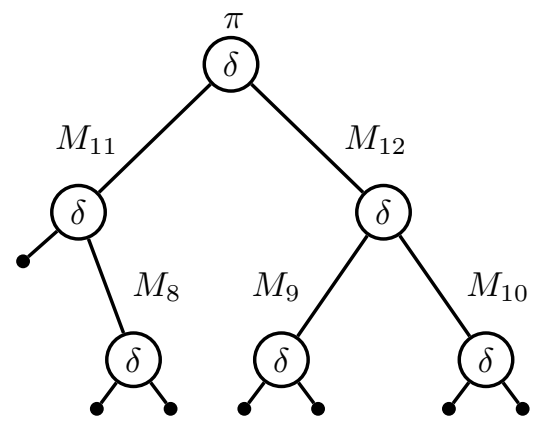

Figure 4: Trimmed phylogenetic tensor $\widetilde{P}$

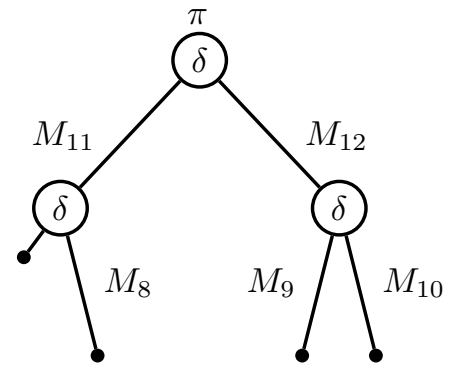

Figure 5: Pruned phylogenetic tensor $P^{*}$

\section{Group representation theory in phylogenetics}

In this section we use the algebraic description of probability distributions on phylogenetic trees given in 2.4 to establish natural connections with aspects of representation theory, for certain groups affiliated to the Markov semigroup. These are discussed in $\$ 3.1$, Then follows $(3.2$ and (3.3) a brief outline of those aspects of the representation theory of the general linear group and its subgroups that are needed for the discussion of group branching rules. This leads to the construction of one-dimensional representations and their identification as invariants (\$3.4), with existence conditions given in 3.5

\subsection{The Markov semigroup and affiliated groups}

The linear transformation (2) effected under the Markov semigroup on probability measures is closely related to certain group actions on the vector space $\mathbb{R}^{k}$. Given that the corresponding representation theory is unchanged [40, in this section we will generalize to complex vector space, (as with [1). That is, here and below, for algebraic purposes we regard the $\delta_{1}, \delta_{2}, \ldots, \delta_{k}$ as elements of a basis for $V \cong \mathbb{C}^{k}$. Thus, the probability measures become a subset lying in the ambient complex space $\otimes^{m} \mathbb{C}^{k} \supset \otimes^{m} \mathcal{M}(K)$. For related considerations involving the study of invariants of stochastic matrices see [38, 53].

Referring to (5) and noting that $-\infty<\operatorname{tr}(Q(t)) \leq 0$ for all $t$, the determinant of each element $M(t, s)$ lies in the interval $(0,1]$, and the Markov semigroup occurs as a subset of the general linear group:

$$
\mathfrak{M}(k) \subset G L(k) .
$$

$G L(k)$ is the group of invertible linear operators on the $k$-dimensional vector space $\mathbb{C}^{k}$. The smallest subgroup of $G L(k)$ that contains $\mathfrak{M}(k)$ is obtained by taking $\mathfrak{M}(k)$ together with all of its operator inverses. In order to apply known methods of representation theory, we will, however, not work with this group directly. We define a slightly less refined subgroup as the focus of the impending discussion.

Generalizing the notation of [53], we define the subgroup $G L_{1}(k) \triangleleft G L(k)$ as the subset of $G L(k)$ 
whose matrices in the $\delta_{1}, \delta_{2}, \ldots, \delta_{k}$ basis have unit column-sum. That is, for all $g \in G L_{1}(k)$ :

$$
\theta^{\top} g=\theta^{\top} .
$$

The group property clearly holds, as for all $g_{1}, g_{2} \in G L_{1}(k)$ :

$$
\theta^{\top}\left(g_{1} g_{2}\right)=\left(\theta^{\top} g_{1}\right) g_{2}=\theta^{\top} g_{2}=\theta^{\top} .
$$

This group is isomorphic to the complex affine group 2

$$
G L(k-1) \ltimes T(k-1) \equiv A(k-1),
$$

where $T(k-1)$ is the group of linear translations on $\mathbb{C}^{k-1}$. As shown in Appendix A this isomorphism is due to the column-sum condition being, in effect, a statement that the group elements are dual to linear transformations in $k$-dimensional complex space, leaving a fixed vector invariant.

Consider also the doubly-stochastic Markov semigroup, $\mathfrak{M}^{*}(k)$, obtained by requiring an additional condition on the rate matrices:

$$
Q(t) \theta=0
$$

The associated subgroup of the general linear group is then denoted as $G L_{1,1}(k)$; the subgroup of matrices in $G L(k)$ which have unit column- and row-sum with, for all $g \in G L_{1,1}(k)$ :

$$
\begin{aligned}
\theta^{\top} g & =\theta^{\top}, \\
g \theta & =\theta .
\end{aligned}
$$

Again the group property can easily be shown to hold. Thus the doubly-stochastic Markov semigroup is naturally affiliated to the associated group $G L_{1,1}(k)$ which, also as shown in Appendix $\mathrm{A}$. itself is isomorphic to $G L(k-1)$.

To summarise, consider the subgroup chain:

$$
G L(k-1) \cong G L_{1,1}(k) \triangleleft G L(k-1) \ltimes T(k-1) \equiv A(k) \cong G L_{1}(k) \triangleleft G L(k) .
$$

and the set inclusions:

$$
\begin{gathered}
\mathfrak{M}(k) \subset G L_{1}(k), \\
\mathfrak{M}^{*}(k) \subset G L_{1,1}(k),
\end{gathered}
$$

We now have a clear picture of how to develop the representation theory of the Markov semigroup which focuses on algebraic properties and avoids the analytic details due to the positivity requirement and semigroup property. This is the correct framework in which to exploit the SchurWeyl duality (\$3.2) and, considering the above inclusions, all results presented will be valid for the Markov semigroup. The above subgroup chain will feature in 3.4 where we give existence conditions for Markov invariants.

\subsection{Representations of $G L(k)$ and Schur-Weyl duality}

Our purpose here is to show that the close relation of the Markov semigroup to affiliated subgroups of the general linear group allows the machinery of representation theory to be applied in analysing the models used in phylogenetic inference.

From the form of the general Markov model on phylogenetic trees given earlier (77), it is evident that the representation-theoretic considerations must be extended to tensor products. We now provide some standard results within this setting.

\footnotetext{
${ }^{2}$ The symbol $\ltimes$ denotes the semi-direct product of two groups 4]. The standard physical example is the Euclidean group, which occurs as the semi-direct product between rotations and translations in $\mathbb{R}^{n}$. These are none other than the set of transformations that define Euclidean geometry.
} 
For $G L(k)$ and its classical subgroups it is well known that for the defining representation on $V \cong \mathbb{C}^{k}$, with $v \mapsto g v$, extended to a reducible representation on $\otimes^{m} V$ in the obvious way, $v_{1} \otimes v_{2} \otimes \ldots \otimes v_{m} \mapsto g v_{1} \otimes g v_{2} \otimes \ldots \otimes g v_{m}$, there is a direct sum decomposition,

$$
\otimes^{m} V=\sum_{\lambda \vdash m} \oplus f_{\lambda} V^{\lambda}
$$

into (possibly reducible) subspaces $V^{\lambda}$. These subspaces (or modules) are labelled by integer partitions, $\lambda=\left(\lambda_{1}, \lambda_{2}, \ldots, \lambda_{n}\right)$, of $m$, the $\lambda_{i}$ being nonzero and nonincreasing and such that $\lambda_{1}+\lambda_{2}+\ldots+\lambda_{n}=m$. If $\lambda$ is a partition of $m$, we write $\lambda \vdash m$ and $|\lambda|=m$. The corresponding module $V^{\lambda}$ is determined by a unique projector on $\otimes^{m} V$; the Young's operator $Y^{\lambda}$. The $f_{\lambda}$ are integer multiplicities determining how many times each module occurs in the decomposition. The Schur-Weyl duality is the classic result that each $f_{\lambda}$ is none other than the dimension of the irreducible representation associated with the same partition $\lambda$ of the symmetric group $\mathfrak{S}_{m}$. This reflects the role of the symmetric group's action on $\otimes^{m} V$ by permuting basis elements across the tensor product, when constructing the Young's operators.

The character of a representation is defined as the set of traces of the representing matrices; one for each group element. The irreducible representations of a group can be enumerated by solely considering the corresponding irreducible characters. Thus the problem of decomposing a representation into irreducible modules (computing the multiplicities $f_{\lambda}$ ) can be performed at the level of the character: 3 .

For instance, in the case of $G L(k)$ itself, the $V^{\lambda}$ are irreducible, with character given by the celebrated Schur functions, $s_{\lambda}$, with

$$
s_{\lambda}(x)=\operatorname{tr}\left(\pi_{\lambda}(g)\right),
$$

where $\pi_{\lambda}(g)$ is the representing matrix for group element $g$ and $x_{1}, x_{2}, \ldots, x_{k}$ are its eigenvalues. The Schur functions are defined in their own right, and are uniquely determined by the semistandard tableaux corresponding to the partition $\lambda[49]$.

The defining $k$-dimensional representation in this notation is $V^{\{1\}} \cong \mathbb{C}^{k}$, in which case the Schur function is $s_{\{1\}}(x)=x_{1}+x_{2}+\ldots+x_{k}$. The Schur functions form a basis for the ring of symmetric functions on any number of variables, and the trace is a symmetric function. Hence, the problem of identifying the irreducible $G L(k)$ modules in the above representation on $\otimes^{m} V$, reduces to identifying the Schur functions in the decomposition of the character with respect to this basis 4 .

A convenient and standard notation for Schur functions is given by enclosing the partition (or parts thereof) in braces [43. Thus $\{\lambda\}$ and $\{1\}$, are the Schur functions corresponding to a general irreducible and the defining representation of $G L(k)$ respectively. For simplicity, we write $\pi_{\{1\}}(g)=g$.

For classical subgroups of $G L(k)$, the modules $V^{\lambda}$ are no longer necessarily irreducible, and further combinatorial considerations (not required here) are needed to effect a complete reduction 5 . More importantly, for $G L(k)$ itself with $V$ not the defining, but an arbitrary module, $V^{\rho}$ say, the equivalents of the above modules, $\left(V^{\rho}\right)^{\lambda}$, are again no longer irreducible in general.

This construction introduces a fundamental operation for combining representations together; that of plethysm [43. The character of $\left(V^{\rho}\right)^{\lambda}$ is denoted $\{\rho\} \underline{\otimes}\{\lambda\}$; the plethysm of $\{\rho\}$ by $\{\lambda\}$. In the simplest case $\{\rho\}$ is the character for the defining representation, $\{1\}$, and by definition $\{1\} \underline{\otimes}\{\lambda\}=\{\lambda\}$.

In general, for any symmetric functions $A, B$ we have $\{\rho\} \underline{\otimes}(A+B)=\{\rho\} \underline{\otimes} A+\{\rho\} \underline{\otimes} B$, and

\footnotetext{
${ }^{3}$ Within the context of phylogenetics, see [50] for an unrelated discussion of the irreducible characters of the symmetric group.

${ }^{4}$ The stronger statement that the $V^{\lambda}$ provide the complete set of irreducible modules of any integral representation of $G L(k)$ is valid [40].

${ }^{5}$ The classical subgroups of $G L(k)$ are constructed by requiring, under the group action, the invariance of bilinear forms on $V$.
} 
we recover

$$
\{\rho\} \underline{\otimes}\left(\sum_{\lambda \vdash m} f_{\lambda}\{\lambda\}\right)=\{\rho\} \otimes\{\rho\} \otimes \ldots \otimes\{\rho\},
$$

where $\{\rho\} \otimes\{\lambda\}$ denotes the (commutative and associative) pointwise multiplication of the Schur functions,

$$
(\{\rho\} \otimes\{\lambda\})(x):=\{\rho\}(x) \cdot\{\lambda\}(x),
$$

and the Schur functions occurring in the decomposition of $\{\rho\} \otimes\{\lambda\}$ correspond to partitions of $|\rho|+|\lambda|$. This of course reflects (10) with $V$ replaced by $V^{\rho}$ :

$$
V^{\rho} \otimes V^{\rho} \otimes \ldots \otimes V^{\rho}=\sum_{\lambda \vdash m} \oplus f_{\lambda}\left(V^{\rho}\right)^{\lambda} .
$$

In particular, for rank 2 we have

$$
V^{\lambda} \otimes V^{\lambda}=\left(V^{\lambda}\right)^{\{2\}} \oplus\left(V^{\lambda}\right)^{\left\{1^{2}\right\}},
$$

which at the level of the characters is described completely by

$$
\{\lambda\} \otimes\{\lambda\}=\{\lambda\} \underline{\otimes}\{2\}+\{\lambda\} \underline{\otimes}\left\{1^{2}\right\} .
$$

This is the well-known decomposition of a representation into its symmetric and anti-symmetric Kronecker square, respectively 6 .

Although it is a difficult task to evaluate the general plethysm (see [49] for a review of symmetric functions and their various manipulations), in practice all required operations of symmetric functions involving products, plethysms and group branching rules can be evaluated symbolically using an appropriate group theory package. Where required, we use Schur [77] for this purpose.

From (7), which gives the form of the phylogenetic tensor for a tree with $m$ leaves, it is clear that the appropriate representation space to consider is indeed $\otimes^{m} \mathbb{C}^{k}$, regarded not as a module of $G L(k)$ as above, but rather carrying an irreducible representation of the action of the direct product group $\times{ }^{m} G L(k)=G L(k) \times G L(k) \times \ldots \times G L(k)$. That is, considering that a phylogenetic tensor lies in the ambient space $\otimes^{m} \mathbb{C}^{k}$, the generic analogue of (7) is

$$
\psi^{\prime}=\left(g_{1} \otimes g_{2} \otimes \ldots \otimes g_{m}\right) \cdot \psi,
$$

where $\psi \in \otimes^{m} \mathbb{C}^{k}$. In a phylogenetic setting, we must allow for differing Markov operators to act on each edge; hence the above form. It is usual in phylogenetics to take a fixed rate-matrix for all edges, and allow the edge lengths to vary, thus creating different Markov operators from identical generators. In fact, the above generalization of the group action allows for differing Markov processes on every edge of the phylogenetic tre 7 .

A complete representation-theoretic analysis incorporating the tree structure of phylogenetic tensors is a topic for future research, and we defer such a theory. We concentrate on analysing the group action defined by (7), leading toward the derivation of Markov invariants while ignoring the underlying tree structure. In $\$ 4$ we will introduce a post-hoc procedure which allows the tree structure to be incorporated. This will allow Markov invariants to be applied in a practical setting without the need for the complete theory.

\footnotetext{
${ }^{6}$ In the context of quantum physics, this corresponds exactly to the statistical properties for ensembles of bosonic and fermionic particles, respectively.

${ }^{7}$ Under a (somewhat biologically unsound) model in which the evolution along the pendant edges occurs with identical transition probabilities, the group becomes the diagonal $G L(k)$ subgroup of the $m$-fold direct product group, and the representation reduces accordingly, precisely as in the initial discussion above.
} 


\subsection{Representations of $\times{ }^{m} G L(k)$}

Here we derive the group branching rule which is required to identify the irreducible modules under the group action (11).

There is yet another product of symmetric functions; the inner product, defined as

$$
\{\lambda\} \odot\{\rho\}=\sum_{\sigma \vdash n} \gamma_{\lambda \rho}^{\sigma}\{\sigma\},
$$

where $|\lambda|=|\rho|=|\sigma|=n$, and the $\gamma_{\lambda \rho}^{\sigma}$ are the integer multiplicities of occurrences of the $\sigma$ representation in the Kronecker product representation between $\lambda$ and $\rho$ of the symmetric group $\mathfrak{S}_{n}$ [4].

Consider the direct product group $G L(k) \times G L(\ell)$, with group action on $V_{1} \otimes V_{2}$, where $V_{1}$ is $k$-dimensional and $V_{2}$ is $\ell$-dimensional, defined by $v_{1} \otimes v_{2} \mapsto g_{1} v_{1} \otimes g_{2} v_{2}$. If the eigenvalues of $g_{1}, g_{2}$ are $x_{1}, x_{2}, \ldots, x_{k}$ and $y_{1}, y_{2}, \ldots, y_{\ell}$ respectively, then the character of this representation is the product

$$
\{1\}(x) \cdot\{1\}(y)=\left(x_{1}+\ldots+x_{k}\right)\left(y_{1}+\ldots+y_{l}\right)=\{1\}(x y),
$$

with

$$
(x y)=\left(x_{1} y_{1}, x_{1} y_{2}, \ldots, x_{2} y_{1}, \ldots, x_{k} y_{\ell}\right) .
$$

Generalizing this result, consider the natural embedding, $G L(k) \times G L(\ell) \subset G L(k \ell)$, and the $\{\lambda\}$ representation of $G L(k \ell)$ restricted to the direct product group: $\Psi \mapsto \pi_{\lambda}\left(g_{1} \times g_{2}\right) \Psi$ with $\Psi \in\left(V_{1} \otimes V_{2}\right)^{\lambda}$. The character of this representation has decomposition

$$
\{\lambda\}(x y)=\sum_{\rho, \sigma \vdash|\lambda|} \gamma_{\rho \sigma}^{\lambda}\{\rho\}(x) \cdot\{\sigma\}(y)
$$

for details see [42, 74. Thus, we see that the inner product plays an essential role in decomposing representations of the direct product group $G L(k) \times G L(\ell)$ into tensor products of irreducible modules of $G L(k)$ with irreducible modules of $G L(\ell)$ :

$$
\left(V_{1} \otimes V_{2}\right)^{\lambda}=\sum_{\rho, \sigma \vdash|\lambda|} \oplus \gamma_{\rho \sigma}^{\lambda} V_{1}^{\rho} \otimes V_{2}^{\sigma} .
$$

Presently we will use this result to derive branching rules for the group action that is relevant to phylogenetics (7).

In its general setting, a (group) branching rule describes the decomposition of a representation of a group, $G$, when restricted to a subgroup, $H \subset G$ (written as $G \downarrow H$ ) [42. For the present purpose, we consider $\times{ }^{m} G L(k)$ as a subgroup of $G L\left(k^{m}\right)$, and given the defining representation of $G L\left(k^{m}\right)$, the corresponding branching rule is

$$
G L\left(k^{m}\right) \downarrow \times^{m} G L(k): \quad\{1\} \longrightarrow\{1\} \otimes\{1\} \otimes \ldots \otimes\{1\}=\otimes^{m}\{1\} .
$$

On the left-side of the arrow, $\{1\}$ denotes the defining representation of $G L\left(k^{m}\right)$, whereas on the right-side, $\{1\}$ denotes the defining representation of $G L(k)$.

If we take the generic $\{\lambda\}$ representation of $G L\left(k^{m}\right)$, the appropriate branching rule is 8

$$
G L\left(k^{m}\right) \downarrow \times{ }^{m} G L(k): \quad\{\lambda\} \longrightarrow \sum_{\sigma_{1}, \sigma_{2}, \ldots, \sigma_{m} \vdash|\lambda|}^{\left\{\sigma_{1}\right\} \odot\left\{\sigma_{2}\right\} \odot \ldots \odot\left\{\sigma_{m}\right\} \ni\{\lambda\}}\left\{\sigma_{1}\right\} \otimes\left\{\sigma_{2}\right\} \otimes \ldots \otimes\left\{\sigma_{m}\right\} .
$$

This result can be confirmed using the identity (12).

\footnotetext{
${ }^{8}$ This is a special case of a more general embedding $\{1\} \rightarrow\left\{\lambda_{1}\right\} \otimes\left\{\lambda_{2}\right\} \otimes \ldots \otimes\left\{\lambda_{m}\right\}$, for which each $\left\{\sigma_{i}\right\}$ in the decomposition is replaced by the appropriate plethysm $\left\{\lambda_{i}\right\} \underline{\otimes}\left\{\sigma_{i}\right\}$. For a recent discussion of the calculus of plethysms see [15].
} 
The branching rule (13) gives the decomposition of irreducible modules of the time evolution at the pendant edges of a tree, as induced by (7), but considered as the representation of $\times{ }^{m} G L(k) \subset$ $G L\left(k^{m}\right)$ defined by

$$
\Psi^{\prime}=\pi_{\lambda}\left(g_{1} \times g_{2} \times \ldots \times g_{m}\right) \cdot \Psi
$$

for $\Psi \in\left(\otimes^{m} \mathbb{C}^{k}\right)^{\lambda}$.

In the setting of phylogenetics, we show in 33.4 that specializing to $\{\lambda\} \equiv\{d\}$ gives the decomposition of (homogeneous degree $d$ ) polynomials of phylogenetic tensors. In a practical setting, this corresponds exactly to taking (polynomial) transformations of the observed data set of character pattern counts. That is, recalling that the expectation value of character pattern counts in a sequence alignment is governed by a joint distribution on a tree corresponding to a phylogenetic tensor $P$, the above branching rule tells us how arbitrary polynomial functions of the character pattern counts decompose into components which transform among themselves under the time evolution given in (77). In addition to what is presented here, this potentially has application to any analysis involving pattern counts in molecular sequence data generally.

In 93.5 , we will exploit the branching rule directly, defining the one-dimensional modules in the decomposition (13) as invariants, and give existence conditions for Markov invariants. We must first establish the isomorphism between homogeneous degree $d$ polynomials on a vector space $V$, and the module $V^{\{d\}}$.

\subsection{Symmetric plethysms and invariants}

Associated with any representation $V$ of a group $G$ is the so-called coordinate $\operatorname{ring} \mathcal{P}(V)$ of polynomials $\sqrt{9}$ over $\mathbb{C}$ in the components $v_{1}, v_{2}, \ldots, v_{k}$, corresponding to a given basis for $V$ [22]. For such polynomials, $f(v)$, there is a natural group action,

$$
f(v) \rightarrow g \cdot f(v):=f\left(g^{-1} v\right) .
$$

There is an isomorphism between the ring $\mathcal{P}(V)$ and the symmetric tensor algebra $10 \vee(V)$ :

$$
\mathcal{P}(V) \equiv \sum_{d=0}^{\infty} \mathcal{P}^{d}(V) \cong \bigvee(V) \equiv \sum_{d=0}^{\infty} \bigvee^{d}(V)
$$

with $\bigvee^{d}(V) \cong V^{\{d\}}$ and $\mathcal{P}^{d}(V)$ denoting the homogeneous polynomials of degree $d$. This reflects that an arbitrary homogeneous polynomial of degree $d$ in $k$ indeterminates can be specified by an array of determinates $f_{i_{1} i_{2} \ldots i_{d}}$ which is symmetric under permutation of indices:

$$
f(v)=\sum_{1 \leq i_{1}, i_{2}, \ldots, i_{d} \leq k} f_{i_{1} i_{2} \ldots i_{d}} v_{i_{1}} v_{i_{2}} \ldots v_{i_{d}} .
$$

Our interest in the above construction lies in the invariant ring, $\mathcal{P}(V)^{G}$, of polynomials that are invariant up to a multiplicative factor under the action of $G$, or more generally, for any subgroup $H \unlhd G$,

$$
f(h v)=\operatorname{det}(h)^{w} f(v),
$$

for all $h \in H$ and $v \in V$. For matrix groups the multiplicative factor is the determinant with $w$ denoting the weight of the invariant. Using the isomorphism (14), the identification of a linear basis of such invariants of degree $d$ reduces to the identification, in the reduction of the $V^{\{d\}}$, of the one-dimensional representations of $H$ in the branching rule $G \downarrow H$.

In particular, the one-dimensional representations of $G L(k)$ occur as follows. Note that the dimension of a representation is equal to the trace of the representing matrix of the identity. For

\footnotetext{
${ }^{9} \mathcal{P}(V)$ is the ring of polynomials in the basis elements, $\xi_{1}, \xi_{2}, \ldots, \xi_{k}$, of the dual space $V^{*}$ so that $\mathcal{P}(V) \equiv$ $\mathbb{C}\left[\xi_{1}, \xi_{2}, \ldots, \xi_{k}\right]$ with $\xi_{i}\left(\delta_{j}\right)=\delta_{i j}$ for all $1 \leq i, j \leq k$.

${ }^{10}$ See [22] for a discussion of the symmetric tensor algebra.
} 
the irreducible module $V^{\lambda}$ this is given by $s_{\lambda}(1,1, \ldots, 1)$. Thus, for a one-dimensional module, the corresponding Schur function must be monomial and (considering the definition of the Schur functions using summations over semi-standard tableaux given in [49) this occurs only for partitions of the form $\left\{r^{k}\right\}$ for any integer $r>0$. Additionally, considering that one-dimensional representations act by simply multiplying by the character itself, and that

$$
\begin{aligned}
s_{\left\{r^{k}\right\}}(x) & =\left(x_{1} x_{2} \ldots x_{k}\right)^{r}, \\
& =\operatorname{det}(g)^{r},
\end{aligned}
$$

we see that, for any $\psi \in V^{\left\{r^{k}\right\}}$, we have

$$
\psi \mapsto \operatorname{det}(g)^{r} \psi,
$$

under the $\left\{r^{k}\right\}$ representation of $G L(k)$. This should be compared directly to (15).

Taking $\mathfrak{M}(k) \subset G L_{1}(k)$, we can construct Markov invariants by identifying polynomials lying in the invariant ring for $G L_{1}(k)$. Clearly, any polynomial

$$
f \in \mathcal{P}\left(\otimes^{m} V\right)^{\times^{m} G L_{1}(k)}
$$

must also satisfy (8) and is hence a Markov invariant. Recalling the salient subgroup chain (9), affiliated to the Markov semigroup, the representation-theoretic task is to evaluate the relevant branching rules for specific cases. The required branching rules derive from (13), together with identification of the specific form of one-dimensional representations of the subgroup in question.

It should be noted that this procedure leaves open the possibility that there exist Markov invariants that do not occur in the invariant ring for $G L_{1}(k)$. We leave this as an open problem, but note that it is plausible that such a possibility could be excluded by continuity arguments.

\subsection{Markov invariants, existence theorems}

Presently, we use the facts we have collected above to establish existence conditions for polynomial invariants for the group actions of $G L(k), G L_{1}(k)$ and $G L_{1,1}(k)$.

Theorem 1: Polynomial invariants for phylogenetic models.

Linearly independent polynomial invariants at degree $d$ of the groups:
i. $\times{ }^{m} G L(k)$,
ii. $\times^{m} G L_{1}(k)$, and
iii. $\times{ }^{m} G L_{1,1}(k)$,

are given by the one-dimensional modules of these groups occurring in the decomposition of the $G L\left(k^{m}\right)$ module $\left(\otimes^{m} V\right)^{\{d\}}$. In each case the one-dimensional modules correspond to $m$-fold products of Schur functions labelled by partitions of $d$ :

i. $\left\{r^{k}\right\} \otimes\left\{r^{k}\right\} \otimes \ldots \otimes\left\{r^{k}\right\}$,

ii. $\left\{r_{1}+s_{1}, r_{1}^{k-1}\right\} \otimes\left\{r_{2}+s_{2}, r_{2}^{k-1}\right\} \otimes \ldots \otimes\left\{r_{m}+s_{m}, r_{m}^{k-1}\right\}$, and

iii. $\left\{r_{1}+s_{1}, r_{1}^{k-2}, t_{1}\right\} \otimes\left\{r_{2}+s_{2}, r_{2}^{k-2}, t_{2}\right\} \otimes \ldots \otimes\left\{r_{m}+s_{m}, r_{m}^{k-2}, t_{m}\right\}$, respectively,

with

$$
\begin{aligned}
& k r \equiv d, \\
& k r_{a}+s_{a} \equiv d, \text { and } \\
& (k-1) r_{b}+t_{b}+s_{b} \equiv d,
\end{aligned}
$$


for all $1 \leq a, b \leq m$ respectively.

Given the isomorphism (14) and the branching rule (13) with $\{\lambda\} \equiv\{d\}$, in each case the number of admissible partitions of the given forms $\left\{\sigma_{1}\right\} \otimes\left\{\sigma_{1}\right\} \otimes \ldots \otimes\left\{\sigma_{m}\right\}$ is the number of times the inner product $\left\{\sigma_{1}\right\} \odot\left\{\sigma_{2}\right\} \odot \ldots \odot\left\{\sigma_{m}\right\}$ of irreducible representations of the symmetric group $\mathfrak{S}_{d}$ contains the one-dimensional irreducible representation $\{d\}$. This is also the number of linearly independent polynomial invariants in each case.

Proof: Each case identifies representations of $\times^{m} G L(k)$ with character $\left\{\sigma_{1}\right\} \otimes\left\{\sigma_{2}\right\} \otimes \ldots \otimes\left\{\sigma_{m}\right\}$, each component of which is a partition that corresponds to a one-dimensional representation of the respective subgroup. The dimension of this representation is the product of the dimension of each of the representations labelled by $\left\{\sigma_{a}\right\}$. Therefore the representation is one-dimensional if and only if, for each $\left\{\sigma_{a}\right\}$, the corresponding representation is one-dimensional.

For case (i), $G L(k)$, as we showed in $\$ 3.4$, the representation labelled by $\left\{r^{k}\right\}$ is one-dimensional, providing an invariant of weight $w \equiv r$. For case (ii), $G L_{1}(k)$, it is established in the appendix that the representation of $G L(k)$ labelled by $\left\{r_{a}+s_{a}, r_{a}^{k-1}\right\}$ contains a unique one-dimensional module under $G L_{1}(k)$. For case (iii), as will also be established in the appendix, $G L_{1,1}(k)$ is isomorphic to $G L(k-1)$ and the $G L(k)$ character $\left\{r_{a}+s_{a}, r_{a}^{k-2}, t_{a}\right\}$ contains under branching to $G L(k-1)$, a unique one-dimensional module with character $\left\{r_{a}^{k-1}\right\}$.

Note that case (ii) is a special instance of case (iii), with $t_{a}=0$, and case (i) is a special instance of case (ii), with $s_{a}=0$. This reflects the definition (15).

Recall the inclusion

$$
\mathfrak{M}(k) \subset G L_{1}(k) \triangleleft G L(k) .
$$

It is clear that any invariant that exists for case (i), with $w \equiv r$, or (ii), with $w \equiv r_{1}=r_{2}=\ldots=r_{m}$, is necessarily a Markov invariant, (8), with the particular form

$$
f(P)=\left(\operatorname{det} M_{1} \operatorname{det} M_{2} \ldots \operatorname{det} M_{m}\right)^{w} f(\widetilde{P}) .
$$

In 4 we will count occurrences of this type of Markov invariant for various cases of interest to phylogenetics; $k=2$ to 4 character states and trees with $m=2$ to 10 leaves. We will also briefly review the algebraic structure of these invariants in the cases $m=2$ to 4 when evaluated upon phylogenetic tensors, and give examples of how this structure can be gainfully employed in the problem of phylogenetic tree reconstruction from molecular sequence data.

Taking case (ii) in its general form, we see that for $w_{1} \equiv r_{1}, w_{2} \equiv r_{2}, \ldots w_{m} \equiv r_{m}$, it is possible that there exist Markov invariants, taking the general form

$$
f(P)=\left(\operatorname{det} M_{1}^{w_{1}} \operatorname{det} M_{2}^{w_{2}} \ldots \operatorname{det} M_{m}^{w_{m}}\right) f(\widetilde{P}) .
$$

When the distinction is required, we refer to these invariants as mixed weight Markov invariants. In 4.3 we will show that such invariants do indeed exist in various cases of interest to phylogenetics. However, as yet the explicit form of these invariants has not been constructed, and their structure remains unexplored.

Recall the inclusion

$$
\mathfrak{M}^{*}(k) \subset G L_{1,1}(k) \subset G L(k),
$$

for the doubly-stochastic Markov semigroup. The case (iii) establishes existence conditions for polynomial invariants for this semigroup. These invariants will be valid for any joint distribution on a phylogenetic tree which is constructed using only doubly-stochastic matrices. This includes oft-used models such as Jukes-Cantor, K80, K3ST and SYM [80]. We report the above theorem, but defer the exploration of the invariants in this case.

\section{Markov invariants in phylogenetics}

In 44.1 we establish existence of Markov invariants relevant to phylogenetics for the cases of $k=2$ to 4 character states, distinguishing between true Markov invariants and invariants which are valid 
for the full general linear group. In 4.2 we report upon known algebraic relations between Markov invariants when evaluated upon phylogenetic tensors for $k=2$ to 4 character states and for trees with $m=2$ to 4 leaves. We also discuss the application of Markov invariants to the problem of phylogenetic tree reconstruction in these cases. Finally, in 4.3 we establish existence of mixed weight Markov invariants for $k=4$ character states and trees with $m=2$ to 5 leaves. Throughout, we used Schur [77] for all non-trivial manipulations of Schur functions.

\subsection{Zoo of invariants and nomenclature}

We gave, in $\widehat{3.4}$, a sufficient condition for the existence of a Markov invariant, (17), of degree $d$ and weight $w$ :

$$
\left\{r+s, r^{k-1}\right\} \odot\left\{r+s, r^{k-1}\right\} \odot \ldots \odot\left\{r+s, r^{k-1}\right\} \ni\{d\},
$$

where $r=w$ and the inner product is taken $m$ times, subsequently written as $\odot^{m}\left\{r+s, r^{k-1}\right\}$.

For reasons discussed below, taking $r=0$ results in the trivial inner product:

$$
\{s\} \odot\{s\}=\{s\},
$$

for all integers $s>0$. Extending to $m>2$,

$$
\odot^{m}\{s\}=\{s\},
$$

and the corresponding Markov invariant is denoted as $\Phi$ with degree $d=1$ and weight $w=0$, and simply expresses the conservation of total probability under the action of the Markov semigroup:

$$
\Phi(P) \equiv \sum_{i_{1}, i_{2}, \ldots, i_{m}} P_{i_{1} i_{2} \ldots i_{m}}=1
$$

Here $\Phi$ is the invariant corresponding to $s=1$ and for $s>1$ the invariant is simply the power $\Phi^{s}$.

For fixed $m$, and any two invariants $f, f^{\prime}$ of degree $d, d^{\prime}$ and weight $w, w^{\prime}$, we can form the pointwise product $f \cdot f^{\prime}$ which is itself an invariant of degree $d+d^{\prime}$ and weight $w+w^{\prime}$. If $w=w^{\prime}$, we can form an invariant from the sum $f+f^{\prime}$. These statements establish that the invariants, $\mathcal{P}(V)^{G}$, form a graded ring [39] (where the grading is over both the degree $d$ and the weights $w$.) In particular, it is important to note that we can increase the degree of any invariant (keeping the weight fixed) by multiplying it with the trivial invariant $\Phi$.

When searching for Markov invariants, we must note that the sufficiency condition (18) will include these powers, and hence in what follows we must allow for this over-counting. In the conclusions we will expand upon this observation with some comments in regard to classifying the ring of invariants.

\section{The general linear, or $s=0$, case}

Recalling Theorem 1, we see that for $s=0$, the Markov invariants are simultaneously invariants under the action of the general linear group. Taking $r=1$, the inner multiplication is trivial:

$$
\left\{1^{k}\right\} \odot\left\{1^{k}\right\}=\{k\} .
$$

This reflects that the Kronecker product of the alternating representation of $\mathfrak{S}_{k}$, associated with the partition $\left(1^{k}\right)$, taken with itself, is the trivial representation, which in turn is associated with the partition $(k)$. Recall that the alternating representation is one-dimensional whose action on $\mathbb{C}$ defined as multiplication by +1 if $\sigma$ is an even permutation and is -1 otherwise. For this one-dimensional representation, the Kronecker product is simply the numeric product, with the result being the trivial representation where every permutation is mapped to +1 . Similarly

$$
\left\{1^{k}\right\} \odot\{k\}=\left\{1^{k}\right\},
$$




\begin{tabular}{|r||r|r|r|r|r|r|}
\hline \multicolumn{1}{|c||}{} & \multicolumn{2}{c|}{$k=2$} & \multicolumn{2}{c|}{$k=3$} & \multicolumn{2}{|c|}{$k=4$} \\
\hline$m$ & $\{21\}$ & $\{31\}$ & $\left\{21^{2}\right\}$ & $\left\{31^{2}\right\}$ & $\left\{21^{3}\right\}$ & $\left\{31^{3}\right\}$ \\
\hline 2 & 1 & 1 & 1 & 1 & 1 & 1 \\
3 & 1 & 1 & 1 & 1 & 0 & 1 \\
4 & 3 & 4 & 4 & 13 & 4 & 16 \\
5 & 5 & 10 & 10 & 61 & 6 & 137 \\
6 & 11 & 31 & 31 & 397 & 40 & 1396 \\
7 & 21 & 91 & 91 & 2317 & 126 & 13881 \\
8 & 43 & 274 & 274 & 14029 & 568 & 138916 \\
9 & 85 & 820 & 820 & 83917 & 2142 & 1388857 \\
10 & 171 & 2461 & 2461 & 504013 & 8824 & 13888996 \\
\hline
\end{tabular}

Table 1: Occurrences of $\{d\}$ in $\odot^{m}\left\{r+s, r^{k-1}\right\}$ with $r k+s=d$

and we see that there exists a single Markov invariant of degree $d=k$ and weight $w=1$ for all even values of $m$.

A very familiar example occurs for $m=2$ where, as we will discuss in 4.2 the invariant arises as the Log-Det distance function [63. In the next case, $m=4$, we refer to the corresponding Markov invariant as the quangle.

Considering $m=2$ and $r=2$, we have

$$
\left\{2^{k}\right\} \odot\left\{2^{k}\right\} \ni\{2 k\},
$$

for $2 \leq k \leq 4$. For each $k$, these invariants can be accounted for by taking the previous invariant and multiplying by $\Phi$. Thus nothing new is gained.

However, taking $m=3$, it follows that there exists an invariant of degree $d=2 k$ and weight $w=2$ :

$$
\left\{2^{k}\right\} \odot\left\{2^{k}\right\} \odot\left\{2^{k}\right\} \ni\{2 k\} .
$$

For $k=2$ this invariant is known in the quantum physics literature as the tangle [13, 14, where it is drawn upon to classify entanglement in 3-qubit systems, and has been generalized for $k=3$ and 4 in the context of phylogenetics in [70. In 4.2 we will briefly review the most striking properties of the tangle relevant to phylogenetics.

\section{Bona-fide Markov invariants, $s>0$}

Here we consider the case $s>0$, where the resulting Markov invariants are not simultaneously valid for the general linear group. In Table 1 we present the number of weight $w=1$ invariants that exist for the cases $k=2,3,4 ; m=2,3, \ldots, 10$ and $s=1,2$. All required computations were performed using Schur, and we have not reduced for over-counting. In Table 2 we summarize the Markov invariants for which we have successfully computed explicit polynomial forms. Here we also record the nomenclature we have developed. Presently we discuss the particular properties of these invariants when evaluated on phylogenetic tensors derived from a tree.

\subsection{What happens on a phylogenetic tree?}

By definition, the expectation value of a (bias corrected) Markov invariant, $f$, depends only upon the internal part of the phylogenetic tree:

$$
E[\widehat{f}(Z)]=f(P)=\left(\operatorname{det} M_{1} \operatorname{det} M_{2} \ldots \operatorname{det} M_{m}\right)^{w} f(\widetilde{P}),
$$

where $Z$ is the observed counts of character patterns, $P$ is the phylogenetic tensor corresponding to the joint distribution on the tree, and the trimmed tensor $\widetilde{P}$, defined in 2.4 , is formed by 


\begin{tabular}{|c|c|c|c|c|}
\hline Name & Symbol & Inner multiplication & Group & $(d, w)$ \\
\hline \multirow[t]{3}{*}{$\operatorname{det}$} & Det & $\odot^{2}\left\{1^{2}\right\}=\{2\}$ & $\times^{2} G L(2)$ & $(2,1)$ \\
\hline & & $\odot^{2}\left\{1^{3}\right\}=\{3\}$ & $\times^{2} G L(3)$ & $(3,1)$ \\
\hline & & $\odot^{2}\left\{1^{4}\right\}=\{4\}$ & $\times{ }^{2} G L(4)$ & $(4,1)$ \\
\hline \multirow[t]{3}{*}{ tangle } & $T$ & $\odot^{3}\left\{2^{2}\right\} \ni\{4\}$ & $\times{ }^{3} G L(2)$ & $(4,2)$ \\
\hline & & $\odot^{3}\left\{2^{3}\right\} \ni\{6\}$ & $\times{ }^{3} G L(3)$ & $(6,2)$ \\
\hline & & $\odot^{3}\left\{2^{4}\right\} \ni\{8\}$ & $\times{ }^{3} G L(4)$ & $(8,2)$ \\
\hline \multirow[t]{3}{*}{ stangle } & $T^{s}$ & $\odot^{3}\{21\} \ni\{3\}$ & $\times{ }^{3} G L_{1}(2)$ & $(3,1)$ \\
\hline & & $\odot^{3}\left\{21^{2}\right\} \ni\{4\}$ & $\times{ }^{3} G L_{1}(3)$ & $(4,1)$ \\
\hline & & $\odot^{3}\left\{31^{3}\right\} \ni\{6\}$ & $\times{ }^{3} G L_{1}(4)$ & $(6,1)$ \\
\hline \multirow[t]{3}{*}{ quangle } & $Q$ & $\odot^{4}\left\{1^{2}\right\} \ni\{2\}$ & $\times^{4} G L(2)$ & $(2,1)$ \\
\hline & & $\odot^{4}\left\{1^{3}\right\} \ni\{3\}$ & $\times{ }^{4} G L(3)$ & $(3,1)$ \\
\hline & & $\odot^{4}\left\{1^{4}\right\} \ni\{4\}$ & $\times{ }^{4} G L(4)$ & $(4,1)$ \\
\hline \multirow[t]{3}{*}{ squangle } & $Q^{s}$ & $\odot^{4}\{21\} \ni 3\{3\}$ & $\times^{4} G L_{1}(2)$ & $(3,1)$ \\
\hline & & $\odot^{4}\left\{21^{2}\right\} \ni 4\{4\}$ & $\times^{4} G L_{1}(3)$ & $(4,1)$ \\
\hline & & $\odot^{4}\left\{21^{3}\right\} \ni 4\{5\}$ & $\times{ }^{4} G L_{1}(4)$ & $(5,1)$ \\
\hline
\end{tabular}

Table 2: Markov invariants of degree $d$ and weight $w$ for $m$ leaves,

setting the lengths of the pendant edges to zero. It is exactly this property that can be exploited in the practical setting of reconstructing phylogenetic trees from molecular sequence data.

As discussed in the closing comments of 93.2 . Markov invariants exist independently of any notion of a tree, and to uncover their potential use in the problem of phylogenetic tree reconstruction it becomes necessary to analyse their structure on particular trees. Crucial to this examination is the generalized pulley principle presented in 70 , which establishes that the family of probability distribution resulting from taking the general Markov model on a particular tree is unchanged under arbitrary placement of the root of the tree (see [1] for an equivalent discussion.) Thus, our task is to search for algebraic relations between the Markov invariants valid for a given $m$, when evaluated upon the trimmed phylogenetic tensors corresponding to particular trees with $m$ leaves. We are free to place the root arbitrarily, and we choose to evaluate the Markov invariants on trees where the root is located to our convenience.

In Appendix B we present the general procedure for computing the explicit polynomial form of Markov invariants using the Young's operators (\$3.2) associated with the relevant partitions. Our general procedure was to take these explicit forms and then search for algebraic relations when the invariants are evaluated on the pruned tensor $\widetilde{P}$ defined by a particular tree. In the general case, any such relations potentially lead to phylogenetically informative statistics, valid under a general model of sequence evolution.

Presently we will report upon this procedure in the known cases, $m=2,3$ and 4 .

\section{The simplest Markov invariant: the Log-Det}

Recall that the generic phylogenetic tensor on $m=2$ leaves (Figure 1) can be written in the form

$$
P=\left(M_{1} \otimes M_{2}\right) \cdot(\delta \cdot \pi) .
$$

The corresponding trimmed tensor, $\widetilde{P}=\delta \cdot \pi$, can be expressed in the $\delta_{1}, \delta_{2}, \ldots, \delta_{k}$ basis with the components

$$
\widetilde{P}_{i_{1} i_{2}}=\delta_{i_{1} i_{2}} \pi_{i_{1}} .
$$

As we showed above, there exists a single Markov invariant for $m=2$. The polynomial form of this invariant is easily derived by considering rank- 2 tensors as matrices, and taking the determinant. Since the invariant is a function on tensors, we make the distinction by using a capital letter and denoting the invariant as Det. This distinction can be compared directly to the use of the determinant function in [5] as opposed to the use in 63. 


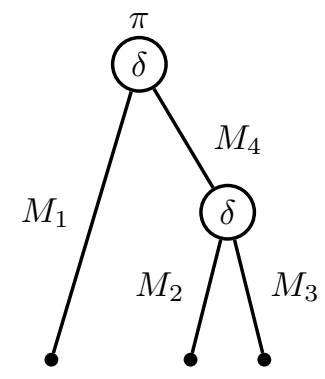

Figure 6: Phylogenetic tensor for the tree $(1,23)$

Substitution gives

$$
\operatorname{Det}(\widetilde{P})=\prod_{1 \leq i \leq k} \pi_{i}
$$

such that, by the definition of Det as a Markov invariant,

$$
\operatorname{Det}(P)=\operatorname{det}\left(M_{1}\right) \operatorname{det}\left(M_{2}\right) \prod_{1 \leq i \leq k} \pi_{i} .
$$

This form holds for any $k$, and is exploited by taking the logarithm and computing the Log-Det distance measure 63 .

\section{Improved pairwise distances: the tangle}

Inspection of Table 1 reveals that for $m=3$ and $s=0$ there exists a Markov invariant, for each of $k=2,3$ and 4 , of degree $d=2 k$ and weight $w=2$. This invariant is valid for phylogenetic trees with three leaves.

The generic phylogenetic tensor on the three leaf tree (Figure 6) can be expressed as

$$
P=\left(1 \otimes M_{2} \otimes M_{3}\right) \cdot(1 \otimes \delta) \cdot\left(M_{1} \otimes M_{4}\right) \cdot(\delta \cdot \pi) .
$$

The trimmed tensor, $\widetilde{P}=(1 \otimes \delta) \cdot\left(1 \otimes M_{4}\right) \cdot(\delta \cdot \pi)$, has components

$$
\widetilde{P}_{i_{1} i_{2} i_{3}}=P_{i_{1} i_{2}}^{*} \delta_{i_{2} i_{3}}
$$

where $P^{*}=\left(1 \otimes M_{4}\right) \cdot(\delta \cdot \pi)$ is the pruned tensor.

The tangle is a Markov invariant and hence satisfies

$$
T(P)=\left(\operatorname{det} M_{1} \operatorname{det} M_{2} \operatorname{det} M_{3}\right)^{2} T(\widetilde{P}) .
$$

By explicit computation (not shown) we have found that

$$
T(\widetilde{P})=\operatorname{Det}^{2}\left(P^{*}\right) .
$$

Thus we see that the induced function of the tangle is $\operatorname{Det}^{2}$. This is the example we promised in \$2.5.

Consistent with (19) we have

$$
\operatorname{Det}\left(P^{*}\right)=\operatorname{det} M_{4}\left(\prod_{1 \leq i \leq k} \pi_{i}\right)
$$

so, finally, we see that

$$
T(P)=\left(\operatorname{det} M_{1} \operatorname{det} M_{2} \operatorname{det} M_{3} \operatorname{det} M_{4}\right)^{2}\left(\prod_{1 \leq i \leq k} \pi_{i}\right)^{2} .
$$


Due to the generalized pulley principle [70, (22) holds for the phylogenetic tensor corresponding to any tree with three leaves. Comparing directly to (19) it is clear that the tangle may be used similarly to the Log-Det pairwise distance but for triplets of molecular sequence data. For further details in this direction see [70].

\section{Informative statistic: the stangle}

We see from Table 1 that for $m=3$ and $s=2$ there also exists, for each $\mathrm{k}=2,3$ and 4 , a weight $w=1$ Markov invariant valid for trees with three leaves (of degree $\mathrm{d}=6$ for $k=4$ states). We refer to this invariant as the stangle, that is, the stochastic tangle.

For the trimmed tensor with components given by (21), explicit computation shows that the stangle satisfies

$$
T^{s}(\widetilde{P}) \equiv 0 .
$$

Thus the stangle is simultaneously a phylogenetic invariant for the general Markov model on a tree with three leaves (of course this again holds for any tree with three leaves.) The stangle has a remarkable coincidence with the phylogenetic invariants presented in [1, and it seems that the existence of the stangle contradicts the conjecture that the ideal of phylogenetic invariants for three taxon trees is generated by the degree $d=5$ and 9 invariants 66.

Given an unbiased estimator, $\widehat{T}^{s}$, of the stangle, we see that under the family of probability distribution described by (20), the expectation value of this estimator when evaluated on triplets of aligned DNA sequences is zero :

$$
E\left[\widehat{T}^{s}(Z)\right]=0,
$$

where $Z$ is the tensor of observed pattern counts in the aligned sequence data. Deviation from zero by the observed value of the stangle, can thus be viewed as evidence that the data set violates the assumptions of the general Markov model. We have had some preliminary (unpublished) success capitalizing on this property to rank subsets of aligned molecular sequences according to apparent concurrence with model assumptions.

\section{Quartet reconstruction: the squangles}

Inspection of Table 1 reveals that for $k=4$ and $m=4$, there exist four Markov invariants of degree $d=5$ and weight $w=1$ relevant to phylogenetic trees with four leaves. We refer to these invariants as the squangles. By explicit computation we have found that three particular linear combinations of the squangles are tree informative. Here we denote these three squangles as $Q_{1}, Q_{2}$ and $Q_{3}$.

On the quartet tree Figure 7 , the generic phylogenetic tensor is

$$
P=\left(M_{1} \otimes M_{2} \otimes M_{3} \otimes M_{4}\right) \cdot(\delta \otimes \delta) \cdot\left(M_{5} \otimes M_{6}\right) \cdot(\delta \cdot \pi) .
$$

The trimmed tensor $\widetilde{P}=(\delta \otimes \delta) \cdot\left(M_{5} \otimes M_{6}\right) \cdot(\delta \cdot \pi)$ has components:

$$
\widetilde{P}_{i_{1} i_{2} i_{3} i_{4}}=P_{i_{1} i_{3}}^{*} \delta_{i_{1} i_{2}} \delta_{i_{3} i_{4}}
$$

with the pruned tensor given by $P^{*}=\left(M_{5} \otimes M_{6}\right) \cdot(\delta \cdot \pi)$. This form of the trimmed tensor can be evaluated directly on the explicit polynomial form of the squangles. We found that on the tree $(12,34)$ the squangles satisfy the algebraic relations:

$$
Q_{1}(\widetilde{P})=0, \quad Q_{2}(\widetilde{P})=-Q_{3}(\widetilde{P})>0,
$$

with, intriguingly, the polynomial form of $Q_{2}(\widetilde{P})$ with respect to the components $P_{i_{1} i_{2}}^{*}$ taking that of the permanen 11, which, unfortunately for the phylogenetic context, is not a Markov invariant.

\footnotetext{
${ }^{11}$ The permanent has identical algebraic form to the determinant of a matrix but with each term positive.
} 


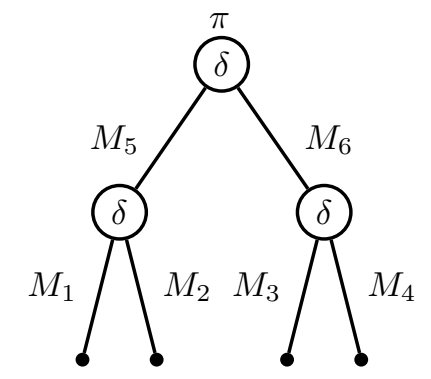

Figure 7: Phylogenetic tensor for the tree $(12,34)$

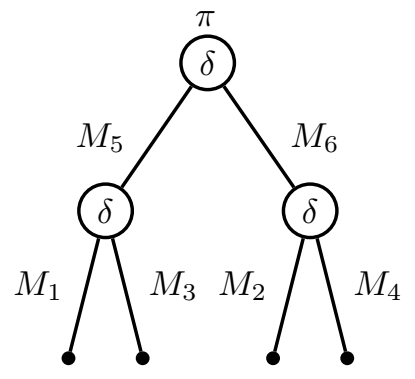

Figure 8: Phylogenetic tensor for the tree $(13,24)$

An identical procedure was carried out on the phylogenetic tensors corresponding to the trees Figure 8 and Figure 9. This produced the relations

$$
\begin{aligned}
Q_{2}(\widetilde{P}) & =0, & Q_{1}(\widetilde{P}) & =Q_{3}(\widetilde{P})>0, \\
Q_{3}(\widetilde{P}) & =0, & -Q_{1}(\widetilde{P}) & =-Q_{2}(\widetilde{P})>0,
\end{aligned}
$$

respectively.

Noting these relations, we see that we have constructed tree-informative phylogenetic invariants for the general Markov model on trees with four leaves. In particular, for the unbiased estimators thereof, we have

$$
E\left[\widehat{Q}_{1}(Z)\right] \equiv 0,
$$

for the tree $(12,34)$,

$$
E\left[\widehat{Q}_{2}(Z)\right] \equiv 0,
$$

for the tree $(13,24)$, and

$$
E\left[\widehat{Q}_{3}(Z)\right] \equiv 0,
$$

for the tree $(14,34)$. We also note that the linear combination

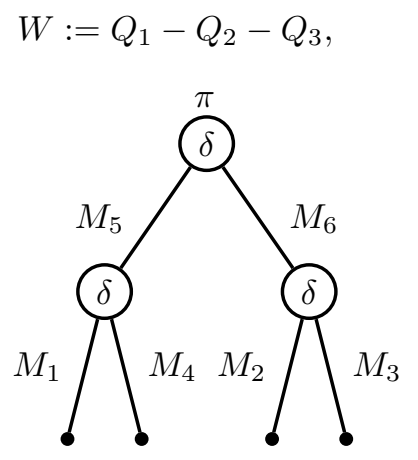

Figure 9: Phylogenetic tensor for the tree $(14,23)$ 
satisfies

$$
E[\widehat{W}(Z)] \equiv 0,
$$

for any phylogenetic tree with four leaves.

With simulation data produced using the Monte Carlo simulation package Hetero [36] under varying input parameters, we have preliminary results suggesting that the squangles are approximately normally distributed (unpublished). Further assuming that the squangles are stochastically independent and that they have identical variance, we have designed a simple quartet inference method optimizing only two parameters, the mean and variance.

The bar charts in Figure 10 compare the success of three tree reconstruction methods tested on data sets created using Hetero. All parameter settings used are as presented in [37] with sequence length $N=1,000$ and 10,000 runs being completed in each case. The three possible quartet trees are $\operatorname{tree} 1=(12,34)$, tree $2=(13,24)$ and tree $3=(14,23)$. For every dataset tree 1 was the true tree. The GC content was made to increase in leaves 1 and 4 and was reduced in leaves 2 and 3 . This tends to bias tree reconstruction to tree 3 as sequences 1 and 4 will tend to be more similar purely because of the GC content.

The Maximum Likelihood and Log-Det + NJ routines were performed using the default settings in Phylip [18, whereas the Markov invariants routine was designed and implemented in $\mathrm{R}$ [58].

While our demonstration is not intended as a fair comparison between the performance of our method and ML using the default settings of Phylip, it does show that using a stationary model for ML can lead to incorrect tree reconstruction if the data was produced by a non-stationary process. With that caveat, it is clear that ML performs very badly as the GC content increases. The Log-Det routine is robust against varying GC content as the technique is based on a general model. This is consistent with what was found in [37. Being valid for the general Markov model, the Markov invariants technique is also robust against varying GC content, and actually performs slightly better than Log-Det.

Interestingly, as the GC content increases, the Log-Det and the Markov invariants infer the true tree more often. Careful inspection reveals that this is because, as the GC content increases, both these techniques tend to infer tree2 less often, rather than tree 3 more often. This effect is more pronounced for the Markov invariants.

\subsection{Mixed weight Markov invariants}

Here we report upon the existence of some mixed weight invariants for various cases of interest to phylogenetics. The polynomial form and algebraic structure on trees of these invariants remains completely unexplored.

We concentrate on $k=4$ and look for mixed weight invariants for the degree $d=8$ partition shapes $\left\{2^{4}\right\}$ and $\left\{51^{3}\right\}$, corresponding to $s=0$ and 4 respectively.

In the $m=2$ case, we find that

$$
\left\{2^{4}\right\} \odot\left\{51^{3}\right\}
$$

does not contain $\{8\}$, which means there does not exist a mixed weight invariant for trees on two leaves.

In the $m=3$ case, we have

$$
\begin{array}{r}
\left\{2^{4}\right\} \odot\left\{51^{3}\right\} \odot\left\{51^{3}\right\} \ni\{8\}, \\
\left\{2^{4}\right\} \odot\left\{2^{4}\right\} \odot\left\{51^{3}\right\} \ni\{8\} .
\end{array}
$$

Writing $w=\left(w_{1}, w_{2}, w_{3}\right)$, we see that, including the three possible permutations across the inner products, there exist mixed weight invariants for the cases $w=(2,1,1),(1,2,1),(1,1,2)$ and $w=$ $(2,2,1),(2,1,2),(1,2,2)$ respectively. 

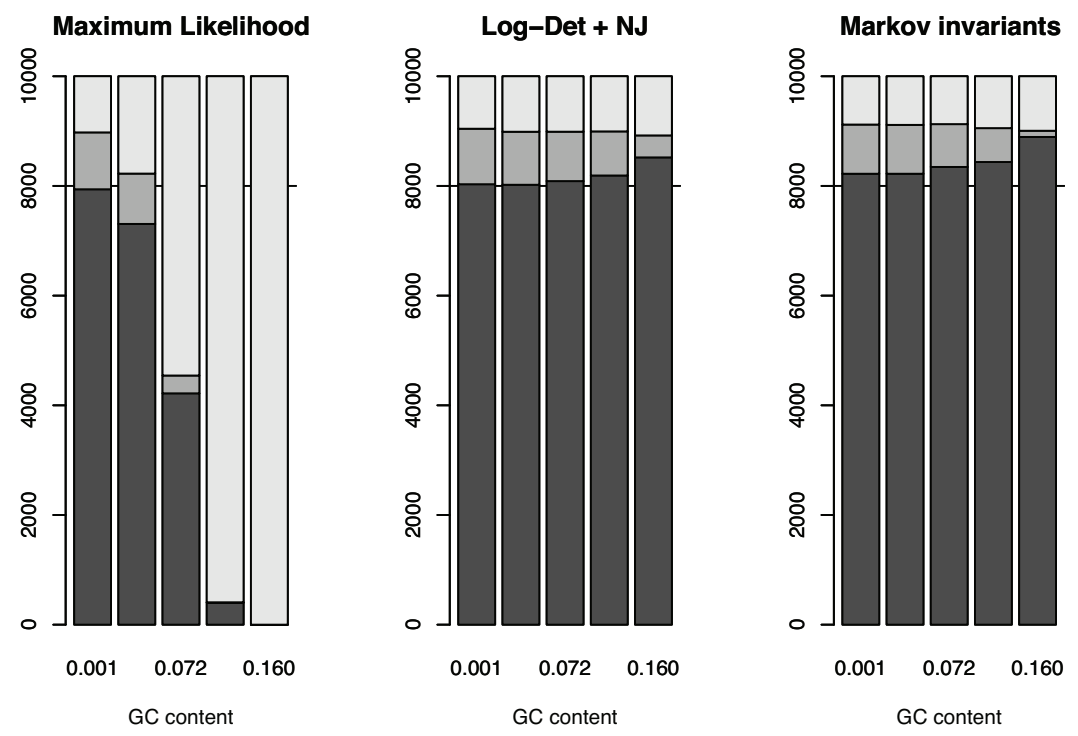

Figure 10: Quartet reconstruction using the squangles. The charts present how many times the tree $1=(12,34)$, tree $2=(13,24)$ and tree $3=(14,23)$ were reconstructed using each of the three methods displayed. The tree used to simulate the data was tree1.

In the $m=4$ case, we have

$$
\begin{array}{r}
\left\{2^{4}\right\} \odot\left\{51^{3}\right\} \odot\left\{51^{3}\right\} \odot\left\{51^{3}\right\} \ni 14\{8\}, \\
\left\{2^{4}\right\} \odot\left\{2^{4}\right\} \odot\left\{51^{3}\right\} \odot\left\{51^{3}\right\} \ni 9\{8\}, \\
\left\{2^{4}\right\} \odot\left\{2^{4}\right\} \odot\left\{2^{4}\right\} \odot\left\{51^{3}\right\} \ni 4\{8\} .
\end{array}
$$

Taking account of the permutations, we see that there exist $14 \times 4=54,9 \times 6=54$ and $4 \times 4=16$ mixed weight invariants for the cases $w=(2,1,1,1), w=(2,2,1,1)$ and $w=(2,2,2,1)$ respectively.

Finally, in the $m=5$ case, we have

$$
\begin{gathered}
\left\{2^{4}\right\} \odot\left\{51^{3}\right\} \odot\left\{51^{3}\right\} \odot\left\{51^{3}\right\} \odot\left\{51^{3}\right\} \ni 527\{8\}, \\
\left\{2^{4}\right\} \odot\left\{2^{4}\right\} \odot\left\{51^{3}\right\} \odot\left\{51^{3}\right\} \odot\left\{51^{3}\right\} \ni 212\{8\}, \\
\left\{2^{4}\right\} \odot\left\{2^{4}\right\} \odot\left\{2^{4}\right\} \odot\left\{51^{3}\right\} \odot\left\{51^{3}\right\} \ni 90\{8\}, \\
\left\{2^{4}\right\} \odot\left\{2^{4}\right\} \odot\left\{2^{4}\right\} \odot\left\{2^{4}\right\} \odot\left\{51^{3}\right\} \ni 46\{8\} .
\end{gathered}
$$

Again taking account of the permutation, we see that there exist $527 \times 5=2635,212 \times 10=2120$, $90 \times 10=900$ and $46 \times 5=230$ mixed weight invariants for the cases $w=(2,1,1,1,1), w=$ $(2,2,1,1,1), w=(2,2,2,1,1)$ and $w=(2,2,2,2,1)$ respectively.

We expect that a future analysis of the explicit form of these invariants will lead to quite an array of informative statistics for phylogenetics.

\section{Discussion}

In this work we have defined and explored the construction of 'Markov invariants'. The primary tool exercised was group representation theory, applied to the usual Markov process present in 
probabilistic models of phylogenetic trees.

It is evident that our present approach to phylogenetics offers many possibilities for further study. The various Markov invariants that we have identified and constructed provide strong candidates for improved tree estimation and parameter recovery under general model assumptions. In particular, the stangle (4.2) seems to provide a robust indicator of phylogenetic signal in subsets of aligned molecular sequences. Efforts are currently underway to incorporate the stangle into a clustering algorithm that provides the means to divide large phylogenetic data sets into smaller, manageable parts. In 4.2 we presented a Markov invariant based quartet reconstruction technique. The optimization we employed was based on rather simple statistical assumptions, and it is clear that this technique could easily be improved upon. For instance, the central limit theorem should provide theoretical justification for taking the invariants as normally distributed and making use of a parametric bootstrap (John Robinson, personal communication, 2007.)

One criticism of the standard phylogenetic invariants is that they have proven to perform poorly with short sequences [28, 30]. An avenue for further research is to establish whether Markov invariants are afflicted by the same curse. We do note that Markov invariants have a rather more constraining definition than the phylogenetic invariants, and, the simplest Markov invariant forms the structure of the Log-Det distance measure which itself is a very popular tool employed in countless phylogenetic studies.

We have shown that Markov invariants occur as one-dimensional representations of a group action associated with the Markov semigroup. In this regard, we applied only a particular instance of the group branching rule (13). It is logical to consider what a similar analysis for irreducible modules of dimension greater than one will reveal with application to phylogenetics. Also, many of the different classes of phylogenetic models [31 can be affiliated with appropriate subgroups of $G L(k)$, and can therefore be expected to have a place in the subgroup chain (9). In principle we can modify Theorem 1 (3.5) for each of these models and construct their associated Markov-type invariants. In this vain, Appendix $\mathrm{C}$ outlines a group-theoretic analysis of the Kimura 3ST model connecting the Hadamard conjugation with the construction of the Cartan subalgebra for this model. The same considerations apply in principle to amino acid sequence models: this is simply a matter of setting $k=20$ and using the same theory, though the computations involved will of course be more lengthy.

As noted in 3.2 a representation-theoretic analysis of the space of phylogenetic tensors that includes the underlying tree structure has not been developed in this work. Ideally, for a given tree, one would like to obtain the structure of the ring of Markov invariants as a theoretical outcome, rather than obtain this structure in the post-hoc procedure presented in 4.2 A possible direction in this regard is to consider, for each tree with $m$ labelled leaves, the subgroup of $\mathfrak{S}_{m}$ induced by identifying permutations that leave the tree and leaf labelling invariant. This subgroup is discussed in Chapter 2 of [59] and, in a different context, in Chapter 3 Topic 12 of [7. We conjecture that this group may play a role, analogous to that of the symmetric group for the Schur-Weyl duality, in the construction of the irreducible modules for the space of phylogenetic tensors.

The phylogenetic invariants form an ideal in the associated polynomial ring, and hence Hilbert's basis theorem for finite-generatedness applies. However, whether Markov invariants are finitelygenerated is unknown. Technically, the group $G L_{1}(k)$ is non-reductive (its finite-dimensional representations are not completely reducible.) In the non-reductive case, standard theorems, such as finite-generatedness of polynomial invariant rings, do not apply. Thus it is unlikely that the Markov type invariants will be finitely generated in general. A notable exception is provided by Weitzenböck's theorem [60, 72] for finite-dimensional representations of one-dimensional Lie groups. Continuing the subgroup chain (9) to its natural limit, it follows that in the case of phylogenetic tensors, the group $G L_{1}(k)$ provides, on restriction, an indecomposable representation of the additive group $\mathbb{R}^{+}$(corresponding to time evolution). Thus, Weitzenböck's theorem is relevant to the analysis of Markov invariants in the current context. In fact, this observation is pertinent to the phylogenetic invariants themselves, as they would occur as syzygies [54 between invariants belonging to the generating set of the invariant ring for the representation of $\mathbb{R}^{+}$in question. 


\section{Acknowledgments}

This paper is the culmination of several years work resulting from interactions with many researchers in what is, for JGS and PDJ, an unfamiliar field. There are many people to acknowledge for their various contributions ranging from simple positive encouragement to important technical insights. In this regard, we would like to thank Michael Baake, Peter Forrester, Alexei Drummond, David Bryant, Mike Steel, David Penny, Mike Hendy, Susan Holmes, Mark Pagel, Andreas Dress, Elizabeth Allman, John Rhodes, John Robinson, Bertfried Fauser, Ron King, Mike Eastwood, Jim Bashford, Malgorzata O'Reilly, and Simon Wotherspoon.

This research was supported by the Australian Research Council grants DP0344996 and DP0770991.

\section{A Proof of Theorem 1}

We provide a tensor-based completion of the proof of Theorem 1 ( 93.5$)$, regarding the identification of one-dimensional irreducible representations of the groups $G L(k), G L_{1}(k)$ and $G L_{1,1}(k)$.

Following the notation of 22.1 a probability measure can be written in a basis of point measures, $\mu=\sum_{1 \leq i \leq k} \mu_{i} \delta_{i}$, with the Markov semigroup acting as

$$
\mu \mapsto M \mu, \quad M \delta_{i}=\sum_{1 \leq j \leq k} \delta_{j} M_{j i}, \quad M \mu=\sum_{1 \leq i \leq k} \mu_{i}^{\prime} \delta_{i}, \quad \mu_{i}^{\prime}=\sum_{1 \leq j \leq k} M_{i j} \mu_{j} .
$$

Moreover, probability conservation requires the column-sum condition

$$
\sum_{1 \leq i \leq k} M_{i j}=1
$$

for all $1 \leq j \leq k$. As discussed in $₫ 3$, this affiliates the linear transformations $M \in \mathfrak{M}(k)$ with the subgroup $G L_{1}(k) \triangleleft G L(k)$. Correspondingly, a higher-rank tensor, $\psi$, transforms under the action of $g \in G L_{1}(k), \psi \mapsto \psi^{\prime}$, with

$$
\psi^{\prime}{ }_{i_{1} i_{2} i_{3} \ldots}=\sum_{1 \leq j_{1}, j_{2}, j_{3} \ldots \leq k} g_{i_{1} j_{1}} g_{i_{2} j_{2}} g_{i_{3} j_{3}} \ldots \psi_{j_{1} j_{2} j_{3} \ldots} .
$$

In order to find combinations of $\psi_{i_{1} i_{2} i_{3} i_{4} \ldots}$ which remain invariant up to scaling under the $G L_{1}(k)$ action, we transform to a more convenient basis in which the distinguished role of the vector $\left(1, e^{\top}\right)=(1,1, \ldots 1)$ is identified. Following [53, define a nonsingular $k \times k$ matrix, $X$, with $1 \times 1+(k-1) \times(k-1)$ block decomposition:

$$
X:=\left(\begin{array}{cc}
1 & e^{\top} \\
\eta & x
\end{array}\right)
$$

Lemma: With respect to the similarity transformation $g \mapsto \widetilde{g}=X g X^{-1}$ defined by any fixed $X$ of the above form, $G L_{1}(k)$ is isomorphic to the affine group $A(k) \cong G L(k-1) \ltimes T(k-1)$. Furthermore, under the same mapping subject to the constraint $\eta=-x \cdot e, G L_{1,1}(k)$ is isomorphic to the group $G L(k-1)$.

Proof: Check explicitly that

$$
\text { if } \quad g=\left(\begin{array}{cc}
\lambda & \ell_{1}^{\top} \\
\ell_{2} & m
\end{array}\right), \quad \text { then } \quad X g X^{-1}=\left(\begin{array}{cc}
1 & 0 \\
\widetilde{\ell} & \widetilde{m}
\end{array}\right),
$$

using the column-sum condition on $g$. Clearly, $\operatorname{det} g=\operatorname{det} \widetilde{m}$, so $\widetilde{m} \in G L(k-1)$ for all such $X$. Finally, if $\ell_{2}=1-m \cdot e, \lambda=1-\ell_{1}^{\top} \cdot e$ and $\eta=-x \cdot e$, then $\tilde{\ell}=0$ in $X g X^{-1}$ and $g \in G L_{1,1}(k)$ is thereby identified with the $G L(k-1)$ subgroup of $G L(k)$ consisting of matrices in block form as displayed. 
It is convenient to re-label the basis as $X \delta_{1}:=\widetilde{\delta}_{0}=\delta_{1}+\delta_{2}+\ldots+\delta_{k}, X \delta_{a}:=\widetilde{\delta}_{a}, a=2,3, \ldots, k$. In the new basis, probability measures will transform inhomogeneously, with the $\widetilde{\delta_{0}}$ components invariant; for example mimicking (A-1)

$$
\widetilde{\mu}_{0}^{\prime}=\widetilde{\mu}_{0}, \quad \widetilde{\mu}_{a}^{\prime}=\widetilde{\ell}_{a} \widetilde{\mu}_{0}+\sum_{b=2}^{k} \widetilde{m}_{a b} \widetilde{\mu}_{b},
$$

and in this way we can deduce the transformation properties of higher-rank tensors.

As we discussed in $\$ 3.2$, the finite dimensional irreducible representations of $G L(k)$ associated with partitions $\lambda$, are realised by tensors of rank $|\lambda|$ whose indices satisfy particular symmetrization conditions to be outlined in Appendix $B$ symmetrize across the rows and then anti-symmetrize down the columns of the associated standard tableau $\mathfrak{T}$. Conventionally, for example, we write for such a tensor the components $\psi_{\left[i_{1} i_{2} \ldots\right]\left[j_{1} j_{2} \ldots\right][\ldots]}$. Here the indices enclosed in braces [...] are mutually anti-symmetric, corresponding to column entries in $\mathfrak{T}$, and there are further cyclic identities (we need not consider) reflecting row dependencies of $\psi$.

Below we will discuss properties of such tensors in the $\widetilde{\delta}_{0}, \widetilde{\delta}_{2}, \ldots \widetilde{\delta}_{k}$ basis under the transformation (A-3). The crucial result will depend absolutely on the indices, and the symbol ' $\psi$ ' will be superfluous. Hence, for ease of reading we will suppress the ' $\psi$ ':

$$
\psi_{\left[i_{1} i_{2} \ldots\right]\left[j_{1} j_{2} \ldots\right][\ldots]} \equiv\left[i_{1} i_{2} \ldots\right]\left[j_{1} j_{2} \ldots\right][\ldots] .
$$

This is consistent with the amusing comments in the preface of [51].

Consider the reduction of an irreducible representation $\lambda$ of $G L(k)$ with respect to the subgroup $G L(k-1)$ (equivalent, by the Lemma above, to considering the restriction to $G L_{1,1}(k)$ affiliated to the doubly-stochastic Markov semigroup.) The partition labels $\bar{\lambda}$ of irreducible representations of $G L(k-1)$ arising from this restriction are related to those of $\lambda$ by the standard betweenness conditions [8, 73, 74]:

$$
\lambda_{1} \geq \bar{\lambda}_{1} \geq \ldots \geq \bar{\lambda}_{n-1} \geq \lambda_{n}
$$

Our present purpose is to identify one-dimensional representations of $G L(k-1)$, that may extend to one-dimensional representations of $G L_{1}(k) \cong G L(k-1) \ltimes T(k-1)$. Recalling (16), such tensor representations must be associated with partitions $\bar{\lambda}=\left(r^{k-1}\right)$ all of whose columns have length $k-1$ corresponding to the $r^{\text {th }}$ power of the representation $M \mapsto \operatorname{det} M$. However, for such a $\bar{\lambda}$, (A-4) above immediately implies that

$$
\lambda=\left(r+s, r^{k-2}, t\right), \quad \text { for some } \quad s \geq 0, t \leq r,
$$

and we have established part (iii) of Theorem 1.

Within such tensor representations of type $\left(r+s, r^{k-2}, t\right)$, the component associated with the scalar representation $\left(r^{k-1}\right)$ of $G L(k-1)$ is clearly

$$
\left[0 a_{12} \ldots a_{1 k}\right]\left[0 a_{22} \ldots a_{2 k}\right] \ldots\left[0 a_{t 2} \ldots a_{t k}\right]\left[b_{11} b_{12} \ldots b_{1, k-1}\right] \ldots\left[b_{r-t, 1} b_{r 2} \ldots b_{r-t, k-1}\right] 0_{1} 0_{2} \ldots 0_{s} .
$$

However, under the inhomogeneous group transformations (A-3) with $\widetilde{m}_{a b}=\delta_{a b}, \widetilde{\ell}_{a} \neq 0$, we have

$$
\begin{gathered}
{\left[0 a_{12} \ldots a_{1 k}\right]\left[0 a_{22} \ldots a_{2 k}\right] \ldots\left[0 a_{t 2} \ldots a_{t k}\right]\left[b_{11} b_{12} \ldots b_{1, k-1}\right] \ldots\left[b_{r-t, 1} b_{r 2} \ldots b_{r-t, k-1}\right] 0_{1} 0_{2} \ldots 0_{s}} \\
\rightarrow \\
{\left[0 a_{12} \ldots a_{1 k}\right]\left[0 a_{22} \ldots a_{2 k}\right] \ldots\left[0 a_{t 2} \ldots a_{t k}\right]\left[b_{11} b_{12} \ldots b_{1, k-1}\right] \ldots\left[b_{r-t, 1} b_{r 2} \ldots b_{r-t, k-1}\right] 0_{1} 0_{2} \ldots 0_{s}+} \\
\widetilde{\ell}_{a_{12}}\left[00 \ldots a_{1 k}\right]\left[0 a_{22} \ldots a_{2 k}\right] \ldots\left[0 a_{t 2} \ldots a_{t k}\right]\left[b_{11} b_{12} \ldots b_{1, k-1}\right] \ldots\left[b_{r-t, 1} b_{r 2} \ldots b_{r-t, k-1}\right] 0_{1} 0_{2} \ldots 0_{s}+\ldots \\
+\widetilde{\ell}_{b_{11}}\left[0 a_{12} \ldots a_{1 k}\right]\left[0 a_{22} \ldots a_{2 k}\right] \ldots\left[0 a_{t 2} \ldots a_{t k}\right]\left[0 b_{12} \ldots b_{1 k-1}\right] \ldots\left[b_{r-t, 1} b_{r 2} \ldots b_{r-t, k-1}\right] 0_{1} 0_{2} \ldots 0_{s} \\
+\widetilde{\ell}_{b_{12}}\left[0 a_{12} \ldots a_{1 k}\right]\left[0 a_{22} \ldots a_{2 k}\right] \ldots\left[0 a_{t 2} \ldots a_{t k}\right]\left[b_{11} \ldots \ldots b_{1, k-1}\right] \ldots\left[b_{r-t, 1} b_{r 2} \ldots b_{r-t, k-1}\right] 0_{1} 0_{2} \ldots 0_{s} \\
+\ldots
\end{gathered}
$$


wherein the coefficients of the $\widetilde{\ell}_{a \ldots}$ terms vanish by anti-symmetry, but those of the $\widetilde{\ell}_{b} \ldots$ terms clearly do not. The components corresponding to the desired $\bar{\lambda}=\left(r^{k-1}\right)$ one-dimensional representation of $G L(k-1)$ within $\lambda=\left(r+s, r^{k-2}, t\right)$ is therefore not invariant under inhomogeneous transformations corresponding to translations in $G L(k-1) \ltimes T(k-1) \cong G L_{1}(k)$ unless the $\left[b_{1,1} \ldots b_{r-t, k-1}\right]$ columns are absent, that is, $t \equiv r$. Thus, the requirement of invariance of the one-dimensional representations under $G L_{1}(k)$ necessitates $\lambda=\left(r+s, r^{k-1}\right)$ as claimed in part (ii) of Theorem 1.

\section{B Young's operators and the construction of Markov in- variants}

We outline the standard construction of the irreducible modules $V^{\lambda}$ as given, for example, in 40.

Consider the representation of $\mathfrak{S}_{m}$ on $\otimes^{m} V$ defined by the action $v_{1} \otimes v_{2} \otimes \ldots v_{m} \mapsto v_{\alpha(1)} \otimes$ $v_{\alpha(2)} \otimes \ldots \otimes v_{\alpha(m)}$ for all $\alpha \in \mathfrak{S}_{m}$. Given a standard tableau $\mathfrak{T}$ with shape $\lambda$ and $|\lambda|=m$, define the permutations $p \in \mathfrak{S}_{m}$ as those that interchange the integers in the same row, and the permutations $q \in \mathfrak{S}_{m}$ as those that interchange numbers in the same column. In the algebra of the representation of the symmetric group whose action is defined above, consider the quantities

$$
A=\sum_{p \in \mathfrak{T}} p
$$

and

$$
B=\sum_{q \in \mathfrak{T}} \operatorname{sign}(q) q .
$$

The Young's operator corresponding to $\mathfrak{T}$ is then defined as

$$
Y^{\lambda}=B A \text {. }
$$

It follows that for a standard tableau of shape $\lambda$, the corresponding Young's operator projects onto an irreducible module of $G L(k)$ :

$$
V^{\lambda}=Y^{\lambda} \cdot \otimes^{m} V
$$

This construction is independent of $k$, and Young's operators corresponding to standard tableau of the same shape project onto equivalent modules. The independent tensor components of these irreducible modules are found by inserting integers from semi-standard tableaux into the indices of the generic tensor. To compute the explicit form of Markov invariants, we must apply this standard procedure to our special case.

Begin with the generic form of a monomial in the components of the tensor $\psi \in \otimes^{m} V$ :

$$
\psi_{i_{1} \ldots i_{m}} \psi_{i_{m+1} \ldots i_{2 m}} \ldots \psi_{i_{m(d-1)+1} \ldots i_{m d}} .
$$

To find the polynomial form of an invariant that arises from an inner product of Schur functions $\left\{\sigma_{1}\right\} \odot\left\{\sigma_{2}\right\} \odot \ldots \odot\left\{\sigma_{m}\right\}$ with $\sigma_{a}=\left\{r+s, r^{k-1}\right\}$ for all $1 \leq a \leq m$, and $r k+s=d$, we must apply the Young's operators to these indices. In an abuse of notation we write

$$
\Psi_{i_{1} \ldots i_{d m}}:=Y^{\sigma_{1}} Y^{\sigma_{2}} \ldots Y^{\sigma_{m}} \psi_{i_{1} \ldots i_{m}} \psi_{i_{m+1} \ldots i_{2 m}} \ldots \psi_{i_{m(d-1)+1} \ldots i_{m d}},
$$

where each Young's operator $Y^{\sigma_{a}}, 1 \leq a \leq m$, is generated from a standard tableau of shape $\left\{\sigma_{a}\right\}$ with integers chosen from the set $\{a, m+a, \ldots,(d-1) m+a\}$. That is, each $Y^{\sigma_{a}}$ permutes the indices $i_{a}, i_{a+m}, \ldots, i_{a+m d}$. The final step is to insert indices into $\Psi$ using the semi-standard tableau which results from filling the $1^{\text {st }}$ row with the integer 0 , and, for $2 \leq i \leq k$, the $i^{\text {th }}$ row with the integer $i$. The justification for filling the "overhang" of length $s$ in the first row of the 
tableau with the integer 0 , is that in the basis given in Appendix $\mathrm{A}$, the $\widetilde{\delta_{0}}$ component is an invariant subspace. For more details, including multiple examples, see [68].

This procedure has been implemented to garner the polynomial form of the Markov invariants for phylogenetic trees with up to four leaves. These are presented in Table 2 , The algorithms required were performed in Mathematica [76, and, unfortunately, do not scale well for trees with more leaves. We are currently investigating the design of efficient algorithms for this construction, and note here that 52 provides a promising direction.

Additionally, in this construction the resulting polynomial form of the invariant is not in the $\delta_{1}, \delta_{2}, \ldots, \delta_{k}$ basis, and the required change of basis computation has thus far not been feasible. To evaluate the invariants on observed data, we therefore proceed by transforming the data itself into the appropriate basis. This allows us to evaluate Markov invariants on observed character pattern counts taken from phylogenetic data sets.

The calculation of unbiased forms, as defined in $\$ 2.2$, is straight forward in principle. However, the calculation requires that the invariants be expressed in the $\delta_{1}, \delta_{2}, \ldots, \delta_{k}$ basis. This appears to be a rather challenging computational task, and to date the required algorithms have not been developed.

\section{Kimura 3ST model and phylogenetic invariants}

Our approach to phylogenetic models via group actions and representations finds specific application in some special cases, such as the Kimura 3ST 41. model and certain generalizations to be described below. Here we provide a brief discussion as an illustration of our focus.

In the usual basis of point measures $\delta_{A}, \delta_{C}, \delta_{U}, \delta_{G}$, the K3ST rate matrix $Q$,

$$
\left[\begin{array}{llll}
Q_{A A} & Q_{A G} & Q_{A U} & Q_{A C} \\
Q_{G A} & Q_{G G} & Q_{G U} & Q_{G C} \\
Q_{U A} & Q_{U G} & Q_{U U} & Q_{U C} \\
Q_{C A} & Q_{C G} & Q_{C U} & Q_{C C}
\end{array}\right]=-(\alpha+\beta+\gamma) 1+\left[\begin{array}{cccc}
0 & \alpha & \beta & \gamma \\
\alpha & 0 & \gamma & \beta \\
\beta & \gamma & 0 & \alpha \\
\gamma & \beta & \alpha & 0
\end{array}\right]
$$

can be re-written [6],

$$
Q=(\alpha+\beta+\gamma)\left(-1+\frac{\alpha}{\alpha+\beta+\gamma} K_{\alpha}+\frac{\beta}{\alpha+\beta+\gamma} K_{\beta}+\frac{\gamma}{\alpha+\beta+\gamma} K_{\gamma}\right),
$$

where the three 'Kimura matrices'

$$
K_{\alpha}=\left[\begin{array}{llll}
0 & 1 & 0 & 0 \\
1 & 0 & 0 & 0 \\
0 & 0 & 0 & 1 \\
0 & 0 & 1 & 0
\end{array}\right], \quad K_{\beta}=\left[\begin{array}{llll}
0 & 0 & 1 & 0 \\
0 & 0 & 0 & 1 \\
1 & 0 & 0 & 0 \\
0 & 1 & 0 & 0
\end{array}\right], \quad K_{\gamma}=\left[\begin{array}{llll}
0 & 0 & 0 & 1 \\
0 & 0 & 1 & 0 \\
0 & 1 & 0 & 0 \\
1 & 0 & 0 & 0
\end{array}\right]
$$

span a Cartan (maximal commuting) subalgebra of the group $S L(4)$, and therefore can be diagonalised simultaneously, via the well-known Hadamard transform [26],

$$
\begin{array}{rl}
H & H=h \otimes h=\left[\begin{array}{cccc}
1 & 1 & 1 & 1 \\
1 & -1 & 1 & -1 \\
1 & 1 & -1 & -1 \\
1 & -1 & -1 & 1
\end{array}\right] \quad, \quad H K_{\alpha} H^{-1}=\left[\begin{array}{cccc}
1 & 0 & 0 & 0 \\
0 & -1 & 0 & 0 \\
0 & 0 & 1 & 0 \\
0 & 0 & 0 & -1
\end{array}\right], \\
H & H K_{\beta} H^{-1}=\left[\begin{array}{cccc}
1 & 0 & 0 & 0 \\
0 & 1 & 0 & 0 \\
0 & 0 & -1 & 0 \\
0 & 0 & 0 & -1
\end{array}\right], \quad H K_{\gamma} H^{-1}=\left[\begin{array}{cccc}
1 & 0 & 0 & 0 \\
0 & -1 & 0 & 0 \\
0 & 0 & -1 & 0 \\
0 & 0 & 0 & 1
\end{array}\right], \\
\text { with } h=\left[\begin{array}{cc}
1 & 1 \\
1 & -1
\end{array}\right] . &
\end{array}
$$


This simple observation means that under this model, rank- $m$ phylogenetic tensors have a spectral resolution given directly in terms of weights of the appropriate $\times^{m}(g l(1) \times g l(1) \times g l(1))$ abelian subalgebra of $\times{ }^{m} G L(4)$ (equivalently the weight decomposition of the corresponding representation of $\left.\times{ }^{m} S L(4)\right)$.

In fact, a stronger statement is possible. The action of group elements of the form $M(t)=e^{t Q}$ turns out to be covariant with respect to the operator $\delta$ introduced in $\$ 2.4$ above, describing branching in the general phylogenetic model - explicitly, in the notation of 2.4 we have

$$
\delta \cdot \exp \left(a K_{\alpha}+b K_{\beta}+c K_{\gamma}\right)=\exp \left(a K_{\alpha} \otimes K_{\alpha}+b K_{\beta} \otimes K_{\beta}+c K_{\gamma} \otimes K_{\gamma}\right) \cdot \delta .
$$

Applied to a phylogenetic tensor $P$ with underlying arbitrary tree $\mathcal{T}$, (A-9) then means that, under this model, the action of the Markov operators on each internal edge can be pulled back to the pendant edges, at the expense of a more complicated edge-mixing transformation. In final form, $P$ is given by the action of a certain element of $(G L(1) \times G L(1) \times G L(1))^{\times m}$ within $G L\left(4^{m}\right)$, with the embedding fixed by the tree, applied to the maximally branched product measure $\delta^{(m-1)} \cdot \pi$, defined by

$$
\delta^{(m-1)} \cdot \pi=\sum_{i} \pi_{i} \delta_{i} \otimes \cdots \otimes \delta_{i},
$$

with $m$ tensor products in each term 12 . Further details can be found in 6 .

Analyses of this sort are useful both analytically, and in explicit calculations. In particular, the identification of phylogenetic invariants for given trees becomes straightforward, once the components of $P$ are written in the diagonal Hadamard basis. The group representation analysis provides a useful alternative to discrete Fourier transform methods which have been successfully applied where rate matrices admit a symmetry with respect to a discrete colour group, $Z_{2} \times Z_{2} \times \cdots$ 26, 27, and may also be useful in the characterisation of phylogenetic varieties in the phylogenetic invariants analysis [3, 67] (see also the discussion in \$5)

The above considerations generalize to the case of any $k$-state model wherein the off-diagonal part of the rate operator is a linear combination of a maximal set of commuting permutation matrices belonging to $\mathfrak{S}_{k}$, which guarantees (A-9). For example, this class would include a 3state model even simpler than the K3ST model, but which is non-symmetric, and for which the Hadamard basis is complex:

$$
\begin{aligned}
Q & =(\alpha+\beta)\left(-1+\frac{\alpha}{\alpha+\beta} K_{\alpha}+\frac{\beta}{\alpha+\beta} K_{\beta}\right), \\
K_{\alpha} & =\left[\begin{array}{lll}
0 & 0 & 1 \\
1 & 0 & 0 \\
0 & 1 & 0
\end{array}\right], \quad K_{\beta}=\left[\begin{array}{lll}
0 & 1 & 0 \\
0 & 0 & 1 \\
1 & 0 & 0
\end{array}\right] .
\end{aligned}
$$

See [6] for further details

\section{References}

[1] E. S. Allman and J. A. Rhodes. Phylogenetic invariants of the general Markov model of sequence mutation. Mathematical Biosciences, 186:113-144, 2003.

[2] E. S. Allman and J. A. Rhodes. Phylogenetic invariants for stationary base composition. Journal of Symbolic Computation, 41:138-150, 2006.

[3] E. S. Allman and J. A. Rhodes. Phylogenetic ideals and varieties for the general Markov model. Advances in Applied Mathematics, to appear, 2007.

[4] A. Baker. Matrix Groups: An Introduction to Lie Group Theory. Springer-Verlag, 2003.

\footnotetext{
${ }^{12}$ This construction can be achieved by noting, for any linear operators $A, B, C, D$ with $A C=C A$ and $B D=D B$, algebraic identities like $e^{A} \otimes e^{B}=e^{(A \otimes 1+1 \otimes B)}$, and $e^{A} \otimes e^{B} \cdot e^{C \otimes D}=e^{(A \otimes 1+1 \otimes B+C \otimes D)}$.
} 
[5] D. Barry and J. A. Hartigan. Asynchronous distance between homologous DNA sequences. Biometrics, 43:261-276, 1987.

[6] J. D. Bashford, P. D. Jarvis, J. G. Sumner, and M. A. Steel. $U(1) \times U(1) \times U(1)$ symmetry of the Kimura 3ST model and phylogenetic branching processes. Journal of Physics A: Mathematical and General, 37:L1-L9, 2004.

[7] L. C. Biedenharn and J. D. Louck. The Racah-Wigner Algebra in Quantum Theory. AddisonWesley, 1981.

[8] L. C. Biedenharn and J. D. Louck. Inhomogeneous basis set of symmetric polynomials defined by tableaux. Proceedings of the National Academy of Sciences USA, 87:1441-1445, 1990.

[9] O. R. P. Bininda-Emonds, editor. Phylogenetic Supertrees: Combining Information to Reveal the Tree of Life. Springer, 2004.

[10] D. Bryant. On the uniqueness of the selection criterion in neighbor-joining. Journal of Classification, 22:3-15, 2005.

[11] K. P. Burnham and D. Anderson. Model Selection and Multi-Model Inference. Springer, 2002.

[12] M. A. Charleston. Hitch-hiking: A parallel heuristic search strategy, applied to the phylogeny problem. Journal of Computational Biology, 8:79-91, 2001.

[13] V. Coffman, J. Kundu, and W. K. Wootters. Distributed entanglement. Physics Review A, 61(5):052306, Apr 2000.

[14] W. Dur, G. Vidal, and J. I. Cirac. Three qubits can be entangled in two inequivalent ways. Physics Review A, 62:062314, 2000.

[15] B. Fauser, P. D. Jarvis, R. C. King, and B. G. Wybourne. New branching rules induced by plethysm. Journal of Physics A: Mathematical and General, 39:2611-2655, 2006.

[16] J. Felsenstein. Cases in which parsimony or compatibility methods will be positively misleading. Systematic Zoology, 27:401-410, 1978.

[17] J. Felsenstein. Inferring Phylogenies. Sinauer Associates, 2004.

[18] J. Felsenstein. PHYLIP (Phylogeny Inference Package) version 3.6. Distributed by the author. Department of Genome Sciences, University of Washington, Seattle., 2005.

[19] O. Gascuel, editor. Mathematics of Evolution and Phylogenetics. Oxford University Press, 2005.

[20] O. Gascuel and M. Steel. Neighbor-joining revealed. Molecular Biology and Evolution, 23:1997-2000, 2006.

[21] G. S. Goodman. An intrinsic time for non-stationary finite Markov chains. Probability Theory and Related Fields, 16:165-180, 1970.

[22] R. Goodman and N. R. Wallach. Representations and Invariants of the Classical Groups. Cambridge University Press, 1998.

[23] X. Gu and W. H. Li. Bias-corrected paralinear and logdet distances and tests of molecular clocks and phylogenies under non-stationary nucleotide frequencies. Molecular Biology and Evolution, 13:1375-1383, 1996.

[24] T. R. Hagedorn. A combinatorial approach to determining phylogenetic invariants for the general model. Tech. Report 2671, CRM, 2000.

[25] P. R. Halmos. Measure Theory. Springer-Verlag, 1974. 
[26] M. D. Hendy and D. Penny. Spectral analysis of phylogenetic data. Journal of Classification, 10:1-20, 1993.

[27] M. D. Hendy, D. Penny, and M. Steel. A discrete Fourier analysis for evolutionary trees. Proceedings of the National Academy of Sciences, 91:3339-3343, 1994.

[28] D. Hillis, J. Huelsenbeck, and D. Swofford. Hobgoblin of phylogenetics? Nature, 369:363-364, 1994.

[29] W. Hordijk and O. Gascuel. Improving the efficiency of SPR moves in phylogenetic tree search methods based on maximum likelihood. Bioinformatics, 21:4338-4347, 2005.

[30] J. P. Huelsenbeck. Performance of phylogenetic methods in simulation. Systematic Biology, 44:17-48, 1995.

[31] J. P. Huelsenbeck, B. Larget, and M. E. Alfaro. Bayesian phylogenetic model selection using reversible jump Markov chain Monte Carlo. Molecular Biology and Evolution, 21:1123-1133, 2004 .

[32] M. Iosifescu. Finite Markov Processes and Their Applications. John Wiley and Sons, Chichester, 1980.

[33] C. Itzykson and J-B. Zuber. Quantum Field Theory. McGraw-Hill, New York, 1980.

[34] P. D. Jarvis, J. D. Bashford, and J. G. Sumner. Path integral formulation and Feynman rules for phylogenetic branching models. Journal of Physics A: Mathematical and General, 38:9621-9647, 2005.

[35] V. Jayaswal, J. Robinson, and L. Jermiin. Estimation of phylogeny and invariant sites under the general Markov model of nucleotide sequence evolution. Systematic Biology, 56:155-162, 2007.

[36] L. S. Jermiin, S. Y. W. Ho, F. Ababneh, J. Robinson, and A. W. D. Larkum. Hetero: A program to simulate the evolution of DNA on four-taxon trees. Applied Bioinformatics, 2:159-163, 2003.

[37] L. S. Jermiin, S. Y. W. Ho, F. Ababneh, J. Robinson, and A. W. D. Larkum. The biasing effect of compositional heterogeneity on phylogenetic estimates may be underestimated. Systematic Biology, 53:638-643, 2004.

[38] J. E. Johnson. Markov-type Lie groups in $G L(n, R)$. Journal of Mathematical Physics, $26: 252-257,1985$.

[39] A. Kelarev. Ring Constructions and Applications. World Scientific, 2002.

[40] R. Keown. An Introduction to Group Representation Theory. Academic Press, New York, 1975.

[41] M. Kimura. Estimation of evolutionary distances between homologous nucleotide sequences. Proceedings of the National Academy of Sciences, 78:1454-1458, 1981.

[42] R. C. King. Branching rules for classical Lie groups using tensor and spinor methods. Journal of Physics A: Mathematical and General, 8:429-449, 1975.

[43] D. E. Littlewood. The Theory of Group Characters. Oxford, Clarendon Press, 1940.

[44] D. E. Littlewood. Plethysm and the inner product of S-functions. Journal of the London Mathematical Society, s1-32:18-22, 1955. 
[45] P. J. Lockhart, A. W. D. Larkum, M. A. Steel, P. J. Waddell, and D. Penny. Evolution of chlorophyll and bacteriochlorophyll: The problem of invariant sites in sequence analysis. Proceedings of the National Academy of Science USA, 93:1930-1943, 1996.

[46] P. J. Lockhart, P. Novis, B. G. Milligan, J. Riden, A. Rambaut, and A. W. D. Larkum. Heterotachy and tree building: A case study with plastids and eubacteria. Molecular Biology and Evolution, pages 40-45, 2006.

[47] P. J. Lockhart, M. A. Steel, A. C. Barbrook, D. H. Huson, and C. J. Howe. A covariotide model describes the evolution of oxygenic photosynthesis. Molecular Biology and Evolution, 15:1183-1188, 1998.

[48] P. J. Lockhart, M. A. Steel, M. D. Hendy, and D. Penny. Recovering evolutionary trees under a more realistic model of sequence evolution. Molecular Biology and Evolution, 11:605-612, 1994.

[49] I. G. MacDonald. Symmetric Functions and Hall Polynomials. Clarendon Press, Oxford, 1979.

[50] F. A. Matsen and S. N. Evans. Ubiquity of synonymity: Almost all large binary trees are not uniquely identified by their spectra or their immanantal polynomials. arXiv:q-bio/0512010, 2006 .

[51] P. McCullagh. Tensor Methods in Statistics. Chapman and Hall, 1987.

[52] A. Molev. On the matrix units for the symmetric group. eprint arXiv:math/0612207, 2007.

[53] B. Mourad. On a Lie-theoretic approach to generalised doubly stochastic matrices and applications. Linear and Multilinear algebra, 52:99-113, 2004.

[54] P. J. Olver. Classical Invariant Theory. Cambridge University Press, Cambridge, 2003.

[55] M. Pagel and A. Meade. A phylogenetic mixture model for detecting pattern-heterogeneity in gene sequence or character-state data. Systematic Biology, 53:571-581, 2004.

[56] D. Penny, B. J. McComish, M. A. Charleston, and M. D. Hendy. Mathematical elegance with biochemical realism: the covarion model of molecular evolution. Journal of Molecular Evolution, 53:711-723, 2001.

[57] D. Posada and T. R. Buckley. Model selection and model averaging in phylogenetics: advantages of Akaike information criterion and Bayesian approaches over likelihood ratio tests. Systematic Biology, 53:793-808, 2004.

[58] R Development Core Team. R: A Language and Environment for Statistical Computing. R Foundation for Statistical Computing, Vienna, Austria, 2006.

[59] C. Semple and M. Steel. Phylogenetics. Oxford Press, 2003.

[60] C. S. Seshadri. On a theorem of Weitzenböck in invariant theory. Journal of Mathematics of Kyoto University, 1:403-409, 1962.

[61] M. Steel. Some statistical aspects of the maximum parsimony method. In R. DeSalle, G. Giribet, and W. Wheeler, editors, Molecular Systematics and Evolution: Theory and Practice., pages 125-140. Birkhäuser Verlag, 2002.

[62] M. Steel. Should phylogenetic models be trying to fit an elephant? Genetics, 21:307-309, 2005.

[63] M. A. Steel. Recovering a tree from the leaf colourations it generates under a Markov model. Applied Mathematics Letters, 7:19-24, 1994. 
[64] M. A. Steel, L. Szekely, P. L. Erdos, and P. Waddell. A complete family of phylogenetic invariants for any number of taxa under Kimura's 3ST model. New Zealand Journal of Botany, 31:289-296, 1993.

[65] K. Strimmer and A. von Haeseler. Quartet puzzling: A quartet maximum likelihood method for reconstructing tree topologies. Molecular Biology and Evolution, 13:964-960, 1996.

[66] B. Sturmfels. Open problems in algebraic statistics. In M. Putinar and S. Sullivant eds., Emerging Applications of Algebraic Geometry, I.M.A. Volumes in Mathematics and its Applications, to appear.

[67] B. Sturmfels and S. Sullivant. Toric ideals of phylogenetic invariants. Journal of Computational Biology, 12:204-228, 2005.

[68] J. G. Sumner. Entanglement, Invariants, and Phylogenetics. PhD thesis, University of Tasmaina, http://eprints.utas.edu.au, 2006.

[69] J. G. Sumner and P. D. Jarvis. Entanglement invariants and phylogenetic branching. Journal of Mathematical Biology, 51:18-36, 2005.

[70] J. G. Sumner and P. D. Jarvis. Using the tangle: A consistent construction of phylogenetic distance matrices. Mathematical Biosciences, 204:49-67, 2006.

[71] C. Tuffley and M. A. Steel. Links between maximum likelihood and maximum parsimony under a simple model of site substitution. Bulletin of Mathematical Biology, 59:581-607, 1997.

[72] R. Weitzenböck. Über die Invarianten von lenearen Gruppen. Acta Mathematica, 58:231-293, 1931.

[73] H. Weyl. The Theory of Groups and Quantum Mechanics. Dover Publications, 1950.

[74] M. L. Whippman. Branching rules for simple Lie groups. Journal of Mathematical Physics, 6:1534-1539, 1965.

[75] M. Wilkinson and J. A. Cotton. Supertree methods for building the tree of life: Divide-andconquer approaches to large phylogenetic problems. In T. R. Hodkinson and J. A. N. Parnell, editors, Reconstructing the Tree of Life: Taxonomy and Systematics of Species Rich Taxa. Systematics Association Special Volume 72. CRC Press, 2006.

[76] Wolfram Research, Inc. Mathematica 5.2. 2005.

[77] B. G. Wybourne. Schur: An interactive programme for calculating properties of Lie groups. version 6.03. http://sourceforge.net/projects/schur, 2004.

[78] Z. Yang. Maximum likelihood phylogenetic estimation from DNA sequences with variable rates over sites: approximate methods. Journal of Molecular Evolution, 39:306-314, 1994.

[79] Z. Yang. Computational Molecular Evolution. Oxford University Press, 2006.

[80] A. Zharkikh. Estimation of evolutionary distance between nucleotide sequences. Journal of Molecular Evolution, 39:315-329, 1994. 